\title{
A benchmark testing technique to characterize the stress-strain relationship in materials based on the spalling test and a photomechanical method
}

\author{
Pascal Forquin ${ }^{1 *}$, Bratislav Lukić ${ }^{1}$, Dominique Saletti ${ }^{1}$, Laurent Sallier ${ }^{2}$, \\ Fabrice Pierron ${ }^{3}$
}

\author{
1 Univ. Grenoble Alpes, CNRS, Grenoble INP ${ }^{* *}$, Laboratoire Sols Solides Structures - Risques \\ (3SR), F-38041, Grenoble, France \\ ${ }^{* *}$ Institute of Engineering Univ. Grenoble Alpes \\ 2 Université de Lorraine, Laboratoire LEM3, F-57000, Metz, France \\ ${ }^{3}$ University of Southampton, Faculty of Engineering and Physical Sciences, SO17 1BJ \\ Southampton, United Kingdom
}

Keywords: Virtual Fields Method, Spalling, Dynamic testing, Concrete, Grid measurement, High-speed imaging

\begin{abstract}
The experimental testing of concrete and other brittle materials under high-strain rate tensile loading remains a major issue in many research fields and industrial applications. Among the experimental methods, the spalling technique provides a major advantage as it does not rely on a static mechanical balance of the sample. A short compressive pulse transmitted to the sample reflects from the rear free surface as a tensile pulse leading to sample tensile fracturing. However, the classic processing (i.e. Novikov's acoustic approximation) based on the measurement of the sample rear face velocity profile, relies on strong assumptions that can lead to an over-estimation of the material tensile strength. A new processing technique was proposed by Pierron and Forquin in 2012 to derive the stress field and apparent Young's modulus using only data from an ultra-high speed camera. This purely inertial method is based on the time resolved measurement of axial displacement fields and the Virtual Fields Methods (VFM). However, the measurement accuracy as well as uncertainty remains to be studied in more detail and better understood. In the present work, a series of spalling tests have been performed with a sample made of aluminium alloy behaving linearly elastic. Such test allows investigating a possible influence of several testing parameters (e.g. interframe time, lens focusing, number of pixels per period...), processing parameters (mainly the levels of spatial and temporal smoothing for regularization) as well as the influence of the camera sensor technology. The results are compared to data from strain gauges and to the expected Young's modulus and stress-strain response of the tested aluminium alloy, so the accuracy of measurements is evaluated. Finally, the spalling set-up applied to a sample of well-known mechanical properties appears to be a good benchmark to assess the quality of stress versus strain measurements based on photomechanical methods.
\end{abstract}

\footnotetext{
* Corresponding author: pascal.forquin@3sr-grenoble.fr, Tel. +33 47682 5248, Fax. +33 476827043
} 


\section{Introduction}

During the last decade, concrete and other rock-like materials have been more and more tested under high strain-rate tension to respond to various demands in the field of safety of concrete structures under accident or attack scenarios [Riedel and Forquin, 2013] or in the field of industrial processes (rock blasting for civil engineering applications, blasting or impact drilling in mining industry...) [Grange et al, 2008; Saadati et al, 2014]. Under such extreme loadings, the geomaterial is successively subjected to high strain-rate confined compression loading (typical hydrostatic pressure about few $\mathrm{MPa}$ ) and tensile loading (typical strain-rates about few hundreds of $1 \mathrm{~s}^{-1}$ ) that produces, respectively, shear deformation and pore collapse due to confining pressure and an anisotropic tensile damage characterised by a high density of oriented cracks [Forquin and Erzar, 2010; Forquin and Hild, 2010]. Several experimental devices are currently used to characterise the response of geomaterials under tensile loading at strain-rates ranging from few $\mathrm{s}^{-1}$ to few hundreds of $\mathrm{s}^{-1}$. On the one hand, Split Hopkinson Bar (SHB) devices are used in tension or in compression to perform, respectively, direct tensile tests [Weerheijm and van Doormal, 2007; Cadoni et al, 2006] or dynamic Brazilian tests (diametric compression of a disc) [Tedesco et al, 1993; Grantham et al, 2004]. In this configuration, quasi-static equilibrium of the concrete sample is assumed to use the impact forces (sample-input bar and sample-output bar) in the processing of the data. However, to achieve this mechanical balance, the loading rate has to be strongly reduced by using for instance pulse-shaping techniques [Frew et al, 2005]. Finally, these techniques remain inadequate to characterise the tensile behaviour of rock-like materials at strain-rates above $10 \mathrm{~s}^{-1}$ [Forquin et al, 2013].

On the other hand, the tensile strength of concrete and geomaterials may be investigated by means of the spalling technique. In such test, the specimen remains in an unbalance state (i.e. no quasi-static equilibrium) as it is loaded by means of a single Hopkinson bar on one end and is free on the opposite end. A striker [Klepaczko and Brara, 2001; Erzar and Forquin, 2010] or a small pyrotechnic charge [Weerheijm and van Doormal, 2007] is generally used to produce a small compression pulse that propagates through the metallic Hopkinson bar and through the sample. It reflects back on the free surface of the sample as a tensile pulse generating the tensile loading leading to the failure of the specimen. This technique formerly developed by Klepaczko and Brara [2001] was improved by Erzar and Forquin [2010] by using an optimized projectile enabling to homogenize the field of tensile stresses in a large part of the sample. However, this technique relies on the assumption of uniaxial stress wave propagation and isotropic linear elastic behaviour up to failure [Forquin et al, 2013; Forquin and Lukić, 2018]. In order to relax these assumptions, Pierron and Forquin [2012] recently proposed to use the images of a high speed camera to measure the displacement field by means of a grid bonded on a plane surface of the sample. Spatial and temporal differentiation enables to derive strain and acceleration maps. The processing of these strain and acceleration maps relied on the use of the Virtual Fields Method [Pierron and Grédiac, 2012]. This photomechanical technique was employed to characterise the tensile strength, the Young's modulus, the stress-strain response of concrete and rocks at high strain-rate [Forquin et al, 2013; Forquin and Lukić, 2018] and the fracture energy of concrete [Lukić et al, 2018]. A recent study has shown that the procedure could be extended to flat composite specimens [Pierron et al, 2014] and ceramic specimens [Fletcher and Pierron, 2018]. In this case, the load was introduced by a direct impact instead of a long bar and strain rates up-to $2000 \mathrm{~s}^{-1}$ were obtained. 
However, the quality of stress and strain measurements and the influence of testing and processing parameters as well as sensors technology still need thorough investigations.

The objective of this article is to propose a benchmark testing method based on the use of spalling technique applied to a sample of well-known behaviour (an aluminium alloy sample) as to firmly assess the influence of testing and processing parameters in order to improve the identification of the stress-strain relationship in view of improving the use of this experimental method to the testing of geomaterials or other types of material. The methodology herein aims to provide a set of optimal testing and processing parameters in the retrieval of the stress-strain material response. Although some basic guidelines on the optimal experimental settings and processing parameters can be obtained through a simulated experiments strategy [Lukić et al, 2017, Van Blitterswyk et al, 2018], only a real experimental condition can faithfully account for the intrinsic noise of both the imaging sensor and the experimental environment. Finally, the proposed benchmark testing method proves to be a useful method of evaluating the performance of an ultra-high speed imaging sensor in the identification of material response.

In the first part, the experimental techniques and the spalling test facility used here are detailed. Then, the basic equations used in the method are reported. Experimental results obtained in experiments performed with an aluminium alloy sample are then analysed through a sensitivity study to investigate the influence of testing parameters (focusing, number of pixels per period, interframe time) and processing parameters (polynomial function of degree, temporal filtering, spatial filtering) and sensor technology.

\section{Test set-up and associated experimental techniques}

\subsection{The spalling testing method}

An experimental set-up has been developed in 3SR Laboratory to perform dynamic tensile tests at high strain-rate. The so-called spalling test consists in a short cylindrical projectile striking a Hopkinson bar for generating an incident compressive pulse that propagates through the Hopkinson bar. The specimen is put in contact with the Hopkinson bar so a part of the incident pulse is transmitted to the sample whereas the other part is reflected in the opposite direction. Next, the transmitted compressive pulse reflects as a tensile pulse on the rear face of the specimen leading to a fast dynamic loading in the core of the sample [Klepaczko and Brara, 2001; Erzar and Forquin, 2010].

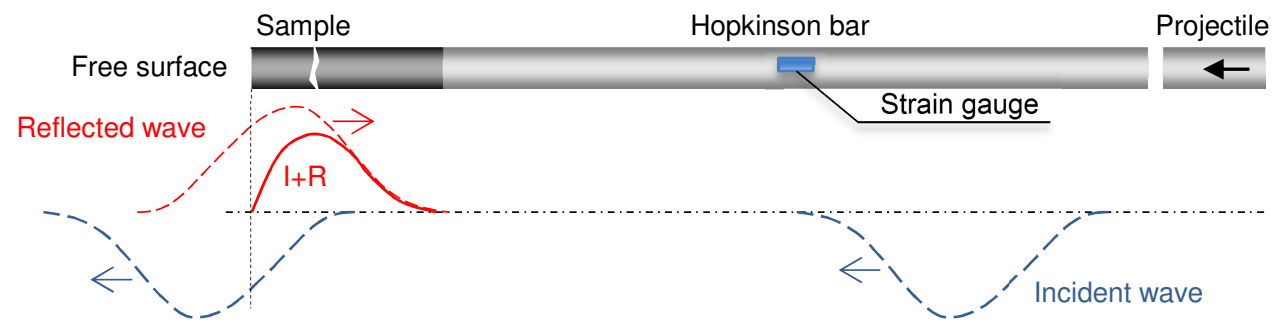

Fig. 1. Schematic of spalling experiments. 
The projectile, the Hopkinson bar and the tested aluminium alloy of diameter $45 \mathrm{~mm}$ are made of the same aluminium alloy. Their density was obtained by weight measurement and the 1D wave speed was characterised by measuring the travelling time of elastic wave though the Hopkinson bar. The aluminium alloy is characterised by a 1D wave speed of $\mathrm{C}=5090 \mathrm{~m} / \mathrm{s}$, a density of $2810 \mathrm{~kg} / \mathrm{m}^{3}$ and a Young's modulus equal to $72.8 \mathrm{GPa}$. The projectile is characterised by a spherical end cap that allows improving the homogeneity of stress in the tested sample [Erzar and Forquin, 2008]. The Hopkinson bar, $1200 \mathrm{~mm}$ in length, is instrumented by a strain-gauge located on its middle cross-section. The tested specimen, $140 \mathrm{~mm}$ in length, is instrumented with 3 strain gauges $10 \mathrm{~mm}$ in length placed at 40,50 and $120 \mathrm{~mm}$ from the rear face. A plane of width equal to the radius of the sample was machined along the sample axis in order to glue the grid on a flat surface (Figure 2). The rear face velocity is recorded with a laser interferometer.

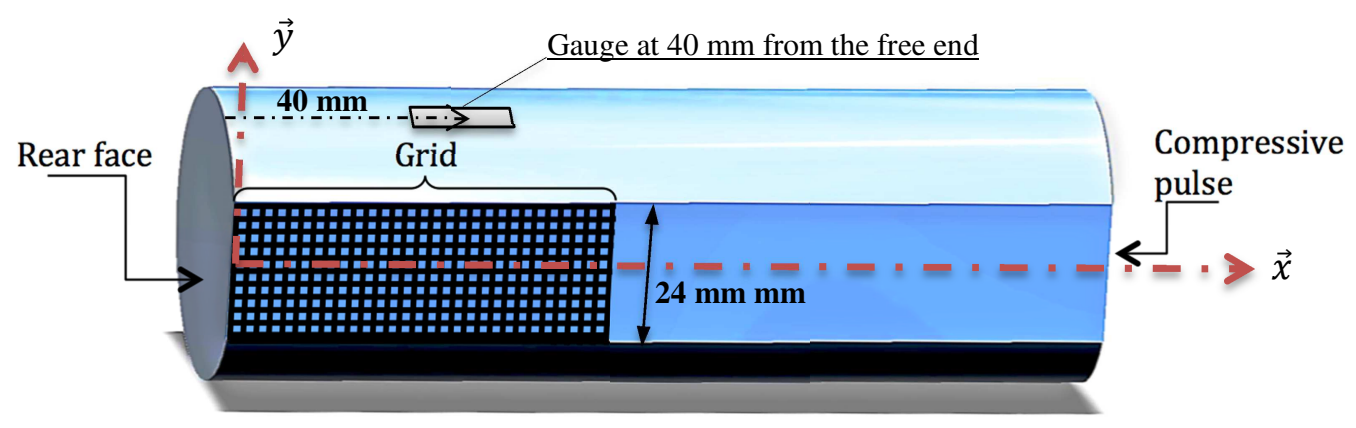

Fig. 2. Schematic of cylindrical sample with flattening on one side.

\subsection{Ultra-high speed cameras}

The first requirement is to acquire images of the patterned specimen using a camera that enables sufficient temporal resolution to allow for accurate acceleration derivation, together with adequate spatial resolution to measure the heterogeneous strain state stemming from the wave propagation. As reported in [Reu and Miller, 2008], there are two families of cameras able to record images at high rates. First, cameras using optimized CMOS sensors for high rate memory read-out. The authors refer to these cameras as 'high speed'. The maximum sampling frequency available at full spatial resolution is around $20 \mathrm{kHz}$. Since the spalling event occurs in a few tens of microseconds, this kind of frame rate is not appropriate here. It is possible to increase the frame rate by compromising the spatial resolution but only to about $100 \mathrm{kHz}$ and with spatial resolutions not adequate to satisfactorily resolve the spatial strain distributions.

The second family is termed 'ultra-high speed' by Reu and Miller [2008]. This encompasses a wide range of technologies based on either rotating mirrors or beam splitting together with the use of many separate CCD sensors so that the memory readout constraint is relieved as each sensor only grabs one image which is read-out after the test. However, these cameras are expensive, difficult to use and present some imaging quality issues, as reported in [Pierron et al, 2011], even though they have been used with some success in the past to record full-field deformations [Kajberg et al, 2004; Moulart et al, 2011; Pierron, Sutton and Tiwari, 2011; Bedsole et al, 2013]. 
Within this family, the Shimadzu HPV-1 camera is based on a dedicated sensor with onchip memory storage capabilities [Etoh et al, 2003]. This technology, also often referred to as in-situ image storage (ISIS), will be used in this work. This acquisition system records images up-to a maximum frequency of $1 \mathrm{MHz}$ with a spatial resolution of 312 by 260 pixels. Some characteristics concerning metrological performance of this sensor and image quality have been examined in detail in [Rossi et al, 2014] and this expertise has been used here to record images of the best possible quality for deformation measurements.

The principle of the HPV-1 camera sensor architecture is described in [Mutoh and Etoh, 2003] (Figs. 3a, 3b, 3c). The sensor is composed of photodiodes (33) connected to the corresponding photo-receptive area (32). Charges are stored in linear CCD charge signal accumulators (36) and transferred to linear CCD charge signal transporters for vertical read-out. Finally, a horizontal readout is made through CCD (39) and charges are amplified and transferred to a buffer memory prior image processing. The microscopic view of the HPV-1 camera sensor (Fig. 3d) highlights the main drawback of the ISIS architecture: most of the space is occupied by the storage unit resulting in a very low fill factor corresponding to the percentage of a pixel devoted to collecting light (around $14 \%$ in the horizontal direction and $76 \%$ in the vertical direction in the Shimadzu HPV-1 sensor, Fig. 3b).
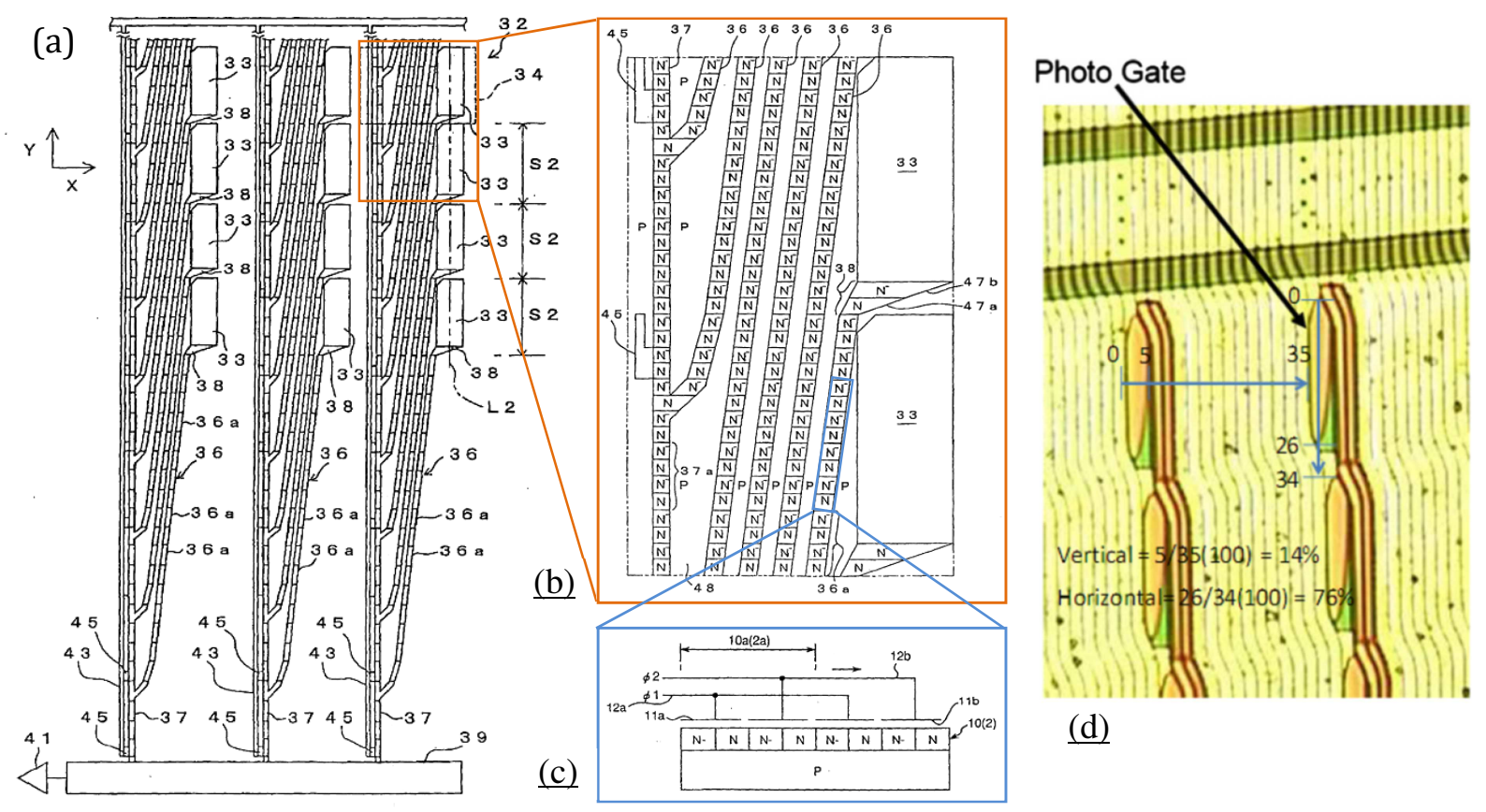

32: photo-receptive area

33: charge signal converter (photodiodes)

36: charge signal accumulator

37: charge signal transporter

Fig. 3 (a) Partial front view showing a photo-receptive area of the high speed image sensor, (b) Partial enlarged front view showing a substrate, (c) Schematic view showing a CCD charge transfer path driven according to a driving voltage having two levels and two phases [Mutot and Etoh, 2003], (d) Microscopic view of the HPV-1 camera sensor [Rossi et al, 2014].

Latest progresses in the domain of ultra-high speed imaging concern the use of CMOS based sensors. These sensors alleviate several limitations of the CCD-based imagers mainly concerning the reduced power consumption and sensor cooling which allowed 
reaching much higher frame rates. One such imaging system that integrates a CMOS imager together with the on-board memory (ISIS) within the pixel domain is the Kirana camera from Specialised Imaging Ltd. [Crooks et al, 2013]. Another imaging system which on the other hand integrates two separate memory arrays outside the pixel domain is the Shimadzu HPV-X2 camera. Both these CMOS based ultra-high speed imagers have been used in this work and are introduced in the following.

The Kirana camera is an ultra-high speed camera based on $u$ CMOS image sensor and circular buffer offering the recording of 180 consecutive frames of 0.7 Megapixel ( $924 \times 768$ pixels) and 10 bit encoding at any recording frame-rates up to $5 \mathrm{Mfps}$. The pixel block diagram is presented in details in Figure 4. It is composed of photodiode distribution consisting of a Vertical Entry bank (VEN) composed of ten cells, ten rows of Lateral banks (LAT) each including 16 cells that are connected to a Vertical Exit bank (VEX) composed of 10 cells. The charges are first read through a single electrode and distributed in the VEN bank. When the VEN is filled charges are pushed laterally to the LAT bank. Finally charges filling the VEX bank can be moved forward to the output structure and the pixel readout circuit.
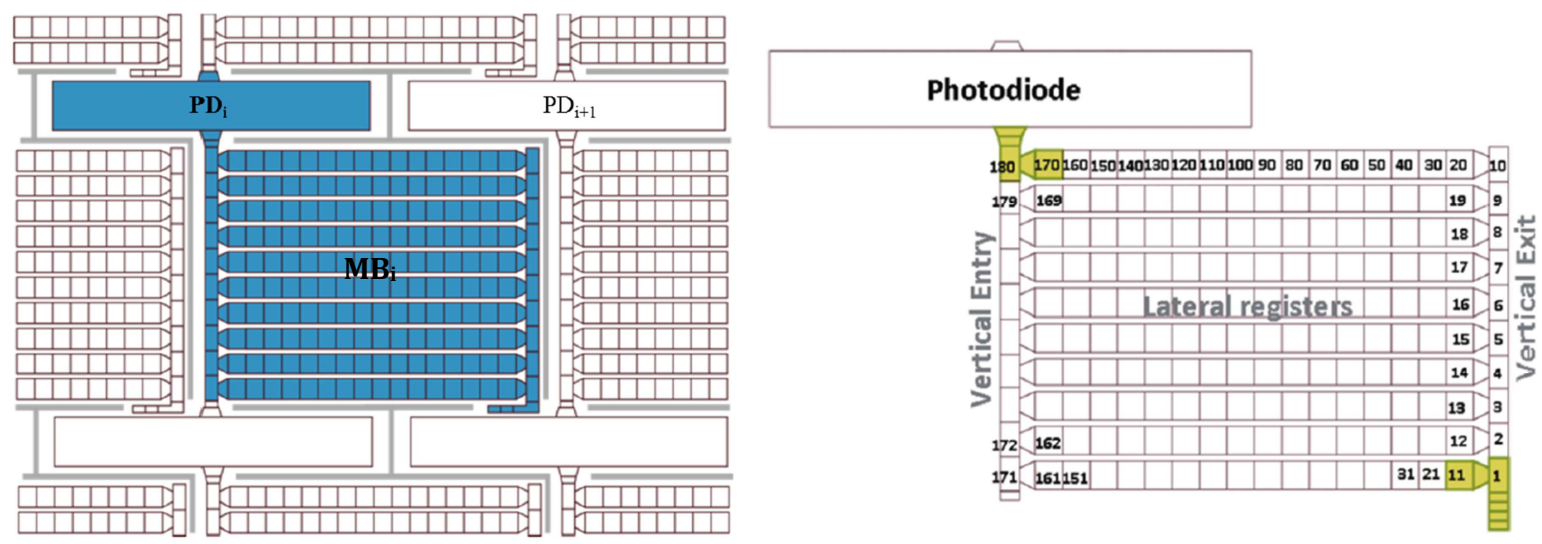

Fig. 4. Pixel block diagram [Crooks et al, 2013a] and layouts of the VEN to LAT and LAT to VEX transfer structures in a Kirana memory bank [Benhammadi et al, 2017]

Shimadzu HPV-X and HPV-X2 are ultra-high speed cameras based on CMOS image sensor technology. Both cameras provide two imaging modes. The HP (half pixel) mode achieves the maximum imaging speed (10 million fps) and allows 256 frames to be recorded with a resolution of about 50,000 pixels by using only two pixels worth of memory for each pixel. In the FP (full pixel) mode, 128 consecutive frames $(400 \times 256$ pixels) are recorded with a maximum recording frequency of $5 \mathrm{Mfps}$. The architecture of the FTCMOS2 sensor (HPV-X2 camera) is based on the same sensor architecture as the HVP-X sensor presented by [Tochigi et al, 2013] but provides eight times higher light sensitivity than the latter due to increased fill factor and increased signal readout gain [Kuroda et al, 2016], though at the cost or two to three times more random noise [Pierron, 2018]. The architecture of the HPV-X2 FTCMOS2 sensor is presented in Figure 5. It is made of two on-chip memory arrays that are separated by the pixel area. The photodiodes in the pixel area and the corresponding memory for recording images are connected by metal wires so that pixel signal outputs are recorded concurrently in memory at high speed.

The noise of Shimadzu HPV-X2 sensor was inspected by means of black image 
acquisition (camera lens completely covered) and blank scene acquisition (illumination of white paper) or acquisition of a gridded surface [Lukić, 2018]. An example of the average grey level of pictures obtained by framing a grid image of a spalling sample with HPV-X2 camera is shown in Figure 6 as a function of the frame count. It is normalised by the camera dynamic range, so it is compared to experimental measurements obtained with the Kirana and the Shimadzu HPV-1 cameras. A stronger temporal flickering effect is noted with the two last sensors compared to the HPV-X2 sensor. The influence of such flickering effect is discussed in the next section.

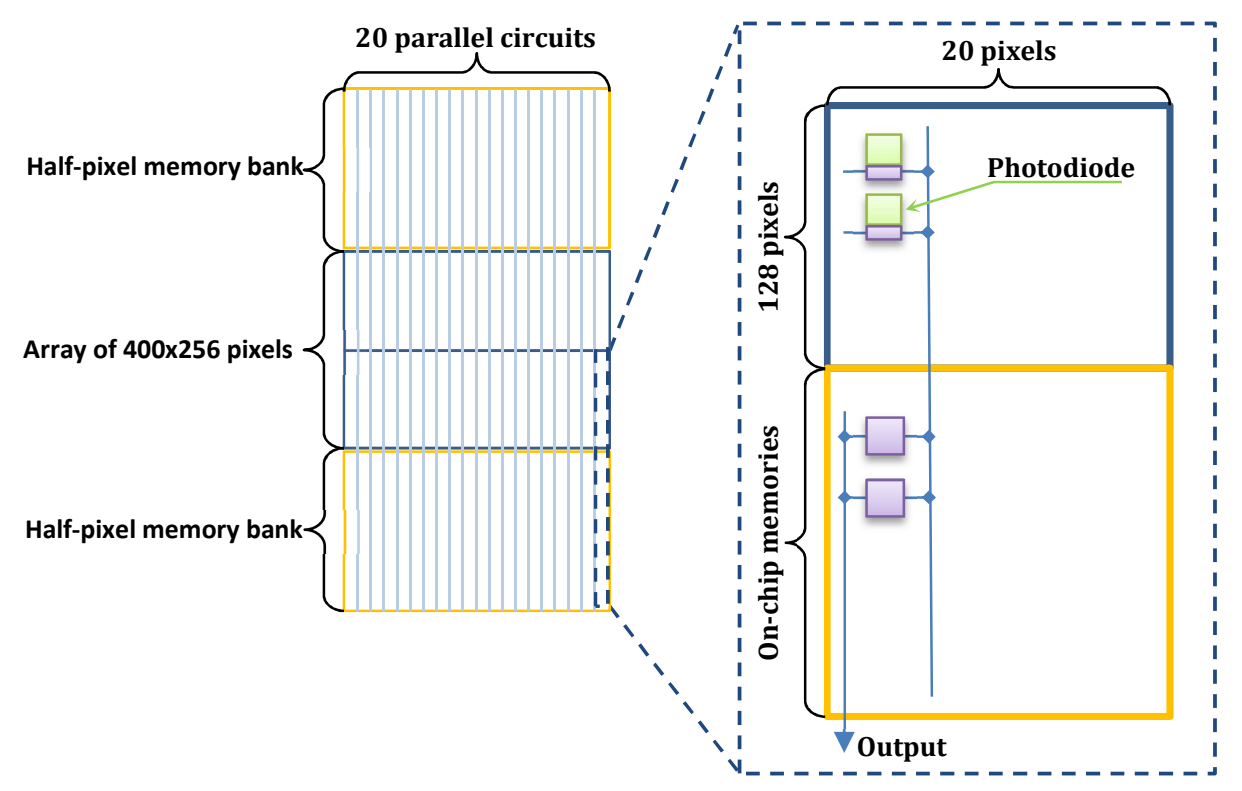

Fig. 5. Schematic diagram of Shimadzu HPV-X2 FTCMOS2 sensor according to [Kuroda et al, 2016].

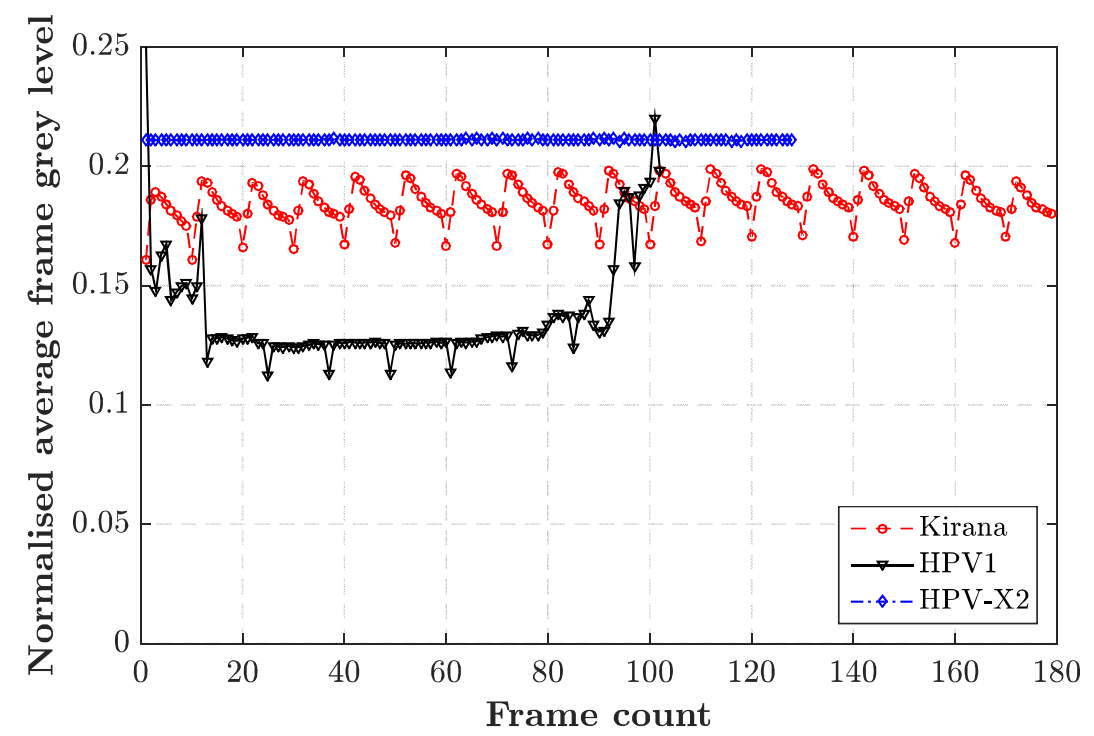

Fig. 6. Average frame grey level normalised by camera dynamic range for three acquired image sequences of the sample grid using the Kirana camera (2 Mfps), the Shimadzu HPV-1 (1 Mfps) and the Shimadzu HPV-X2 camera (2 Mfps). 


\subsection{Full-field time resolved displacement measurements}

The data processing technique relies on the availability of full-field time resolved displacements. In the existing literature, this is mostly achieved in dynamics by a combination of high speed imaging and random pattern image processing though digital image correlation [Sutton et al, 2009] or the use of a regular pattern (the Grid Method) enabling the processing of the images with phase-shifting techniques [Grédiac et al, 2016].

Digital Image Correlation (DIC) is certainly the most widely used full-field deformation measurement technique. It has been employed by many research groups in the past to successfully capture high speed deformation fields, mostly with high speed cameras, see for instance [Grantham et al, 2004; Gilat et al, 2009; Peirs et al, 2011], sometimes in stereo mode using two cameras [Besnard et al, 2012] and sometimes with UHS cameras [Kajberg et al, 2004; Luo et al, 2006; Bedsole et al, 2013]. However, one of the main limitations of DIC is its poorer displacement resolution to spatial resolution compromise compared to the Grid Method [Grédiac et al, 2017].

An alternative consists in using a regular pattern instead of a random one. The use of a regular grid enables the processing of the images with phase-shifting techniques to obtain displacements. This achieves an independent displacement measurement point for every subset of typically $5 \times 5$ pixel $^{2}$, which leads to at least ten times more data points than with DIC, for about the same displacement resolution ( 0.01 pixel). In the past, this technique, called the Grid Method, has been used in quasi-static (see [Surrel 1994; Badulescu et al, 2011; Avril et al, 2004], among others) but has found a particularly interesting application at high rates to mitigate the generally lower camera spatial resolutions [Pierron and Forquin, 2012; Moulart et al, 2011; Pierron et al, 2014; van Blitterswyk et al, 2018; Fletcher and Pierron, 2018; Seghir and Pierron, 2018]. It is beyond the scope of the present paper to detail the Grid Method, the reader is referred to a recent review of the method provided in [Grédiac et al. 2016]. The basic working principle relies on the use of a spatial phase-shifting algorithm to extract spatial phase from the grey level grid images, from which the displacement is obtained by multiplying the phase by $\mathrm{p} / 2 \pi$, where $\mathrm{p}$ is the pitch of the grid.

A grid has been bonded onto the specimen using the procedure developed by [Piro and Grédiac, 2004], as shown in Fig. 2. Sequences of images of this grid were captured during the tests as to produce displacement maps as a function of time. The grid pitch, the grid sampling and the frame-rate considering for each camera are detailed in the Table 1.

Table 1. Testing parameters for three high-speed cameras

\begin{tabular}{llll}
\hline Camera & Shimadzu HPV-1 & Kirana & $\begin{array}{l}\text { Shimadzu HPV- } \\
\text { X2 }\end{array}$ \\
\cline { 1 - 2 } $\begin{array}{l}\text { Full-field technique } \\
\text { measurement }\end{array}$ & Grid & Grid & Grid \\
Sensor size (pixels) & $312 \times 260$ & $924 \times 768$ & $400 \times 250$ \\
Lens & Nikon $105 \mathrm{~mm}$ & Sigma $105 \mathrm{~mm}$ & Sigma $105 \mathrm{~mm}$ \\
& $\mathrm{f} / 2.8$ & $\mathrm{f} / 2.8$ Macro & f/2.8 Macro \\
Total number of images & 102 & 180 & 128
\end{tabular}


Grid sampling (in pixels)

Grid pitch (in mm)

Frame rate (Mfps)
4,5

1

$0.5,1$
13

1

2
7

1

2

Diffuse approximation ( $2^{\text {nd }}$ order) [Avril et al, 2010] used to improve the strain resolution with a radius of 8 data points $(8 \mathrm{~mm})$

\section{Principle of the Virtual Fields Method (VFM)}

From the time resolved strain and acceleration maps, the objective is to extract information about the mechanical behaviour of the materials. There are several techniques available to do this, as reviewed in [Avril et al., 2008]. Finite Element Model Updating (FEMU) is the usual choice to solve this problem; however, it is very costly numerically because of the necessity to run a large number of finite element calculations to minimize a distance between experiment and model [Kajberg et al., 2004]. Here, the Virtual Fields Method (VFM) has been used to process the data.

The VFM relies on the principle of the virtual work which can be written as follows:

$$
-\int_{V} \sigma: \varepsilon^{*} d V+\int_{\partial V} T \cdot u^{*} d S=\int_{V} \rho a \cdot u^{*} d V
$$

where $\sigma$ is the stress tensor, $\varepsilon^{*}$ the virtual strain tensor associated to $u^{*}, T$ the stress vector at the boundary of the solid $\partial V, u^{*}$ the virtual displacement vector, $\rho$ is the density, $a$ the acceleration vector and ' $'$ ' and ' ' represent the scalar product between second order tensors and vectors respectively. The idea is to write this equation with specific virtual fields to derive integral equilibrium equations. The stress terms are then substituted using the constitutive equations and the integrals approximated by discrete sums provided that the test is $2 \mathrm{D}$ as no measurements are available in the bulk. In the case of linear elastic behaviour, this leads to solving simple linear systems to obtain elastic stiffness components [Pierron and Grédiac, 2012].

There are many ways to use this equation, as reported in [Pierron and Grédiac, 2012]. In the past [Pierron and Forquin, 2012], this particular problem has been approached in two different ways, which correspond to two particular choices of virtual fields. First, it is possible to easily extract Young's modulus by assuming that the stress state is uniaxial (but not uniform obviously, as stress waves travel along the test specimen). This is done by using the following virtual fields (with axes as on Fig. 2):

$$
\left\{\begin{array} { c } 
{ u _ { x } ^ { * } = f ( x ) } \\
{ u _ { y } ^ { * } = 0 }
\end{array} \quad \left\{\begin{array}{c}
\varepsilon_{x}^{*}=f^{\prime}(x) \\
\varepsilon_{y}^{*}=0 \\
\varepsilon_{s}^{*}=0
\end{array},\right.\right.
$$

where $f$ is a continuous and differentiable function of $y$, with $f(0)=0$ to avoid having a contribution of the stress vector at the boundary of the field view where stresses exist. This function has to be selected so that it 'follows' the displacement field. To do so, the actual longitudinal displacement maps have been used to construct $f(x)$. First, these maps have been averaged over the width to provide a 1D function, which has then been expended in the transverse direction so that $\mathrm{f}$ only depends on $x$. Then, a constant value 
has been added to ensure that $f(x=\mathrm{L})=0$. The derivative of $f(x)$ was then simply computed by through the coefficients of the fitted polynomial function. After some simple derivations reported in [Pierron and Forquin, 2012], a simple expression of the Young's modulus $\left(E_{V F M}\right)$ is obtained at each time step of the test:

$$
E_{V F M}=\rho \frac{\overline{a_{x} f(x)}}{\overline{\varepsilon_{x} f^{\prime}(x)}}
$$

where the over bar signifies the spatial average of the quantity under it over the field of view.

The second approach uses a rigid-body like virtual field to compute average stresses in each cross section of the specimen:

$$
\left\{\begin{array} { l } 
{ u _ { x } ^ { * } = 1 } \\
{ u _ { y } ^ { * } = 0 }
\end{array} \quad \left\{\begin{array}{l}
\varepsilon_{x}^{*}=0 \\
\varepsilon_{y}^{*}=0 \\
\varepsilon_{s}^{*}=0
\end{array}\right.\right.
$$

Denoting $\sigma_{x}(x, t)$ the average of the axial stress in the section over the $y$ direction, Equation (1) becomes:

$$
0+S \sigma_{x}(x, t)=-S \rho x \overline{a_{x}(x, t)}
$$

where $\overline{a_{x}(x, t)}$ is the spatial average of the longitudinal acceleration component over the surface lying between the free end and the considered section of coordinate $y$. Again, the detailed derivation can be found in [Pierron and Forquin, 2012]. Finally:

$$
\sigma_{x}(x, t)=-\rho x \overline{a_{x}(x, t)}
$$

Since strain maps are also available, it is possible from this equation to plot stressstrain curves in each cross-section of the field of view. This approach, somewhat similar to that suggested by [Aloui et al., 2008], has also been successfully employed in [Pierron et al., 2014]. The rest of the paper is dedicated to the presentation of the results and the sensitivity analysis.

4. Experimental results obtained with an aluminium alloy sample and a Shimadzu HPV-1 ultra-high speed camera

A series of experiments have been conducted on aluminium samples instrumented with strain gauges and a grid of $1 \mathrm{~mm}$ pitch. The sample and the Hopkinson bar being from the same aluminium alloy, the sample's Young's modulus was deduced by measuring the density $(\rho)$ and the one-dimensional wave speed $\left(C_{0}\right)$ in the Hopkinson bar according to the formula:

$$
E_{\text {alu }}=\rho\left(C_{0}\right)^{2}
$$

This measurement that relies on the assumption of $1 \mathrm{D}$ wave propagation is made possible thanks to the very low wave dispersion in the $1.2 \mathrm{~m}$ long Hopkinson bar that results from the compressive pulse shaping obtained by using a hemispheric smoothend projectile providing typical rising time of more than $30 \mu \mathrm{s}$ as detailed in [Erzar and Forquin, 2010; 2011].

The reference experiment, named Alu6, was conducted with clear focusing (no blurring), at the maximum recording frequency available with the HPV-1 Shimadzu 
camera (1 Mfps) and with magnification such that 5 pixels sampled each grid period (the number of pixels being 312 in the $x$ direction the length of the field of view is $62.4 \mathrm{~mm}$ ). The results are presented hereafter.

\section{Measurement of apparent Young's modulus with an aluminium sample}

Full-field displacement measurements for the Alu6 experiment have been first processed to evaluate the "apparent Young's modulus" based on Equation (3). However, the quality of measurement depends on the noise on the acceleration and the strain fields resulting from, respectively, the double temporal and the single spatial differentiations of the displacement field. In order to reduce the influence of noise in the experimental data and retrieve the expected Young's modulus, a filtering procedure has been used. In the time domain, a polynomial function of varying degree denoted ' $n$ ' is considered to fit the curve of axial displacement as function of time. The temporal filtering is applied in the form of a sliding window over a number of consecutive frames denoted ' $l$ '. In the same way, a second degree polynomial fit over a spatial window size of 8 points was considered to calculate the strain.

The obtained result in terms of "apparent Young's modulus" versus time in the Alu6 experiment is detailed in Figure 7 with the parameters $(n=2, l=11)$. It is observed that in the compression part of the loading, when the mean acceleration level is high enough (Figure 7b), the Young modulus of the aluminium sample seems to be correctly evaluated (Figure 7a). For instance, between $\mathrm{T}=195 \mu$ s and $\mathrm{T}=215 \mu \mathrm{s}$, the 'apparent Young modulus' varies from $68 \mathrm{GPa}$ to $99 \mathrm{MPa}$ compared to the expected value of Young's modulus equals to $72.8 \mathrm{GPa}$. On the other hand, in the tensile part of the loading, the 'apparent Young modulus' is not very well evaluated. The main reason is coming from the low amplitude of stresses especially near the free end. Finally, this method does not seem to be reliable enough to obtain the stiffness of the material. This is most probably caused by a non-optimal choice of the virtual fields in Eq.(3) but also by measurement bias and uncertainties propagating into the identification procedure. Here, the virtual fields were obtained by differentiating a $10^{\text {th }}$ degree polynomial, fitted in a least-squared sense to the averaged displacement field at each time step. More adapted procedures based on noise-optimized virtual fields have shown to provide better stiffness identification data [Avril et al, 2004; van Blitterswyk et al, 2018]. In any case, this paper focuses mainly on the supposedly more promising second method based on stress reconstruction in the cross-section which is evaluated in more depth in the following considering different processing and testing parameters. 


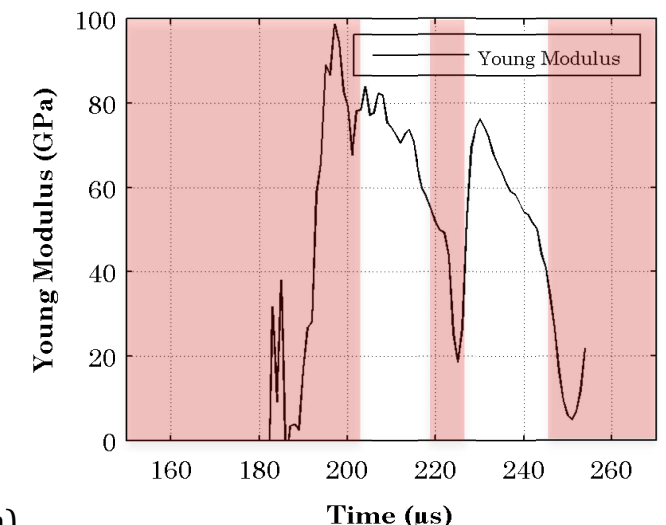

(a)
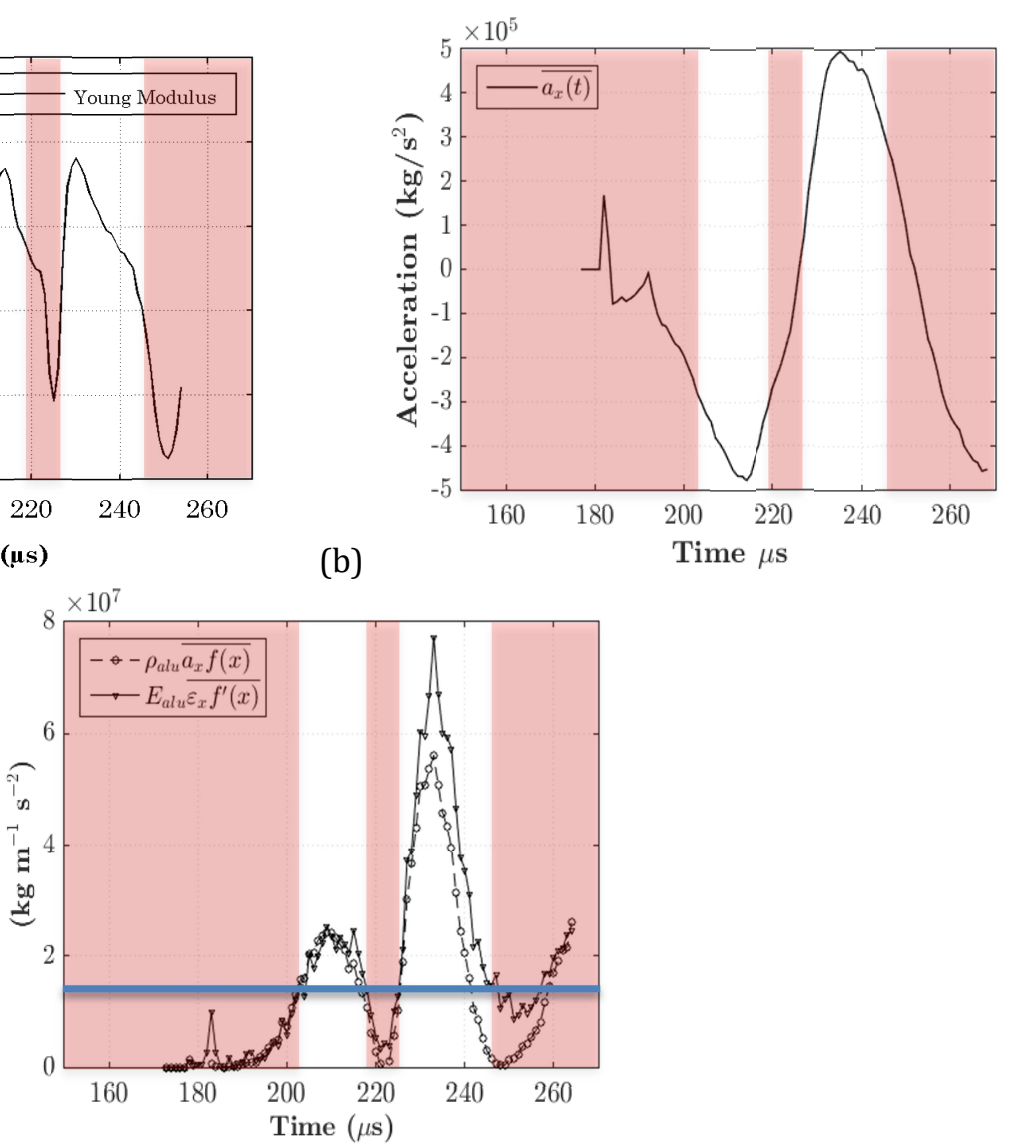

(c)

Fig. 7. Processed data for test Alu6 (clear focusing, 1 Mfps, 5 pixels per period, $n=2, l=11$ ). (a) Young's modulus $\left(E_{V F M}\right)$ versus time (Equation (3)), (b) averaged acceleration over the field of view as a function of time, (c) numerator and denominator of the Equation (3) considering expected value of material parameters. The pink bands arbitrely define the zones where the denominator of Equation (3) is less than $2.10^{-4}\left(E_{a l u} \overline{\varepsilon_{x} f^{\prime}(x)} \leq 1.45 \times 10^{7} \mathrm{~kg} / \mathrm{m} / \mathrm{s}^{2}\right)$.

\section{$\underline{\text { Identification based on stress reconstruction from acceleration }}$}

The second method based on the stress reconstruction from acceleration is now evaluated. The reference experiment, named Alu6, is considered again (clear focusing, $1 \mathrm{Mfps}, 5$ pixels per period). Again a smoothing procedure has been applied: first, in the time domain, a polynomial function of degree ' $n$ ' $(n=2)$ is considered to fit the curve of axial displacement as function of time, using a sliding window over ' $l$ ' consecutive frames; then the stress given by equation 6 is obtained from the derived acceleration. . Finally, in the space domain, the axial strain is averaged along a length denoted 'virtual gauge size'. Three spatial sizes of virtual gauge have been considered in the present work as presented a bit further.

The results obtained by processing the Alu6 experimental data are detailed in Figure 8. First, the strain in the cross-section at $40 \mathrm{~mm}$ from the free surface is plotted as function of time in Figure 8a. The curve obtained by averaging the grid strains over the equivalent gauge area is compared to the data from the strain gauge. A very good correlation is observed between both measurements. Second, the stress level in the cross-section at $40 \mathrm{~mm}$ from the free surface calculated from Equation 6 is plotted as function of time in Figure $8 \mathrm{~b}$ by considering the following parameters in the processing program $(n=2, l=11$, virtual gauge size $=10 \mathrm{~mm})$. Compression in the cross-section is 
observed in the first $40 \mu \mathrm{s}$. Then, the loading reverses to tension. The stress-strain curve in the cross-section at $40 \mathrm{~mm}$ from the free surface is plotted in Figure 8c. A linear elastic behaviour is observed in the considered range of stress and strain, even though a significant amount of noise is present because of the low strain levels. A maximum error may be estimated from the difference in stress and strain levels between the fitted straight line (Young's modulus of $72.8 \mathrm{GPa}$ ) and the data. The error does not exceed about $\pm 7 \mathrm{MPa}$ regarding the stress level and $\pm 1.10^{-4}$ regarding the strain level, which is comparable to the measurement noise floor in strain. Linear regression is performed to retrieve material stiffness, considering both the first compressive and further tensile stages of the loading at three characteristic distances from the sample free end $(30,40$ and $50 \mathrm{~mm}$ ). The results regarding the retrieved Young's Modulus $\left(E_{\text {lin. }}\right)$ calculated by performing a linear regression of the stress-strain curve and the standard deviation of the stress residuals $\left(\sigma_{r e s}^{e s t}\right)$ are provided in Table 2, the standard deviation being defined as:

$$
\sigma_{\text {res }}^{e s t}=\sqrt{\frac{1}{n} \sum_{i=1}^{n}\left(\Delta_{i}-\bar{\Delta}_{i}\right)^{2}} \text { with } \Delta_{i}=\sigma_{i}-E \varepsilon_{i} \text { and } \bar{\Delta}_{i}=\frac{1}{n} \sum_{i=1}^{n} \Delta_{i},
$$

where $E$ is the Young's modulus of the aluminium alloy (72.8 GPa), $\sigma_{i}$ are the stress points (Equation (6)) and $\varepsilon_{i}$ are the measured strain points.

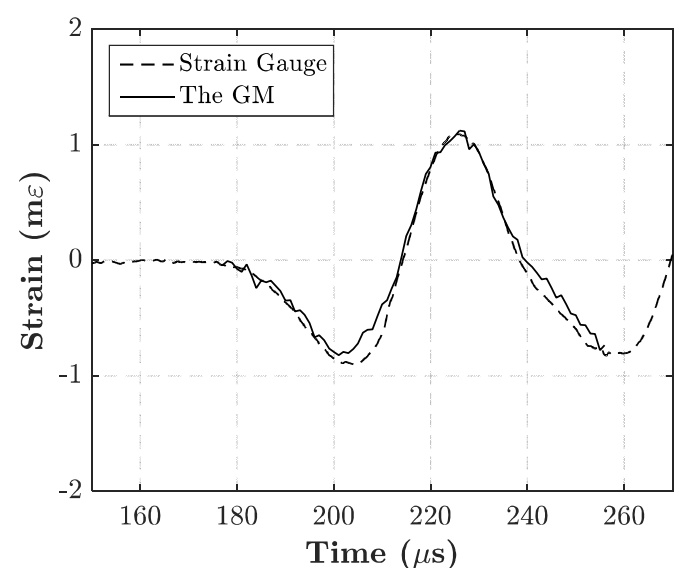

(a)

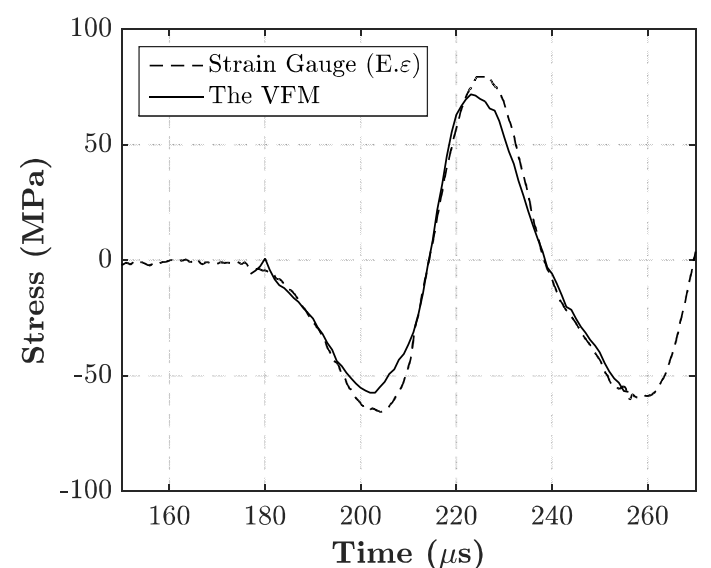

(b)

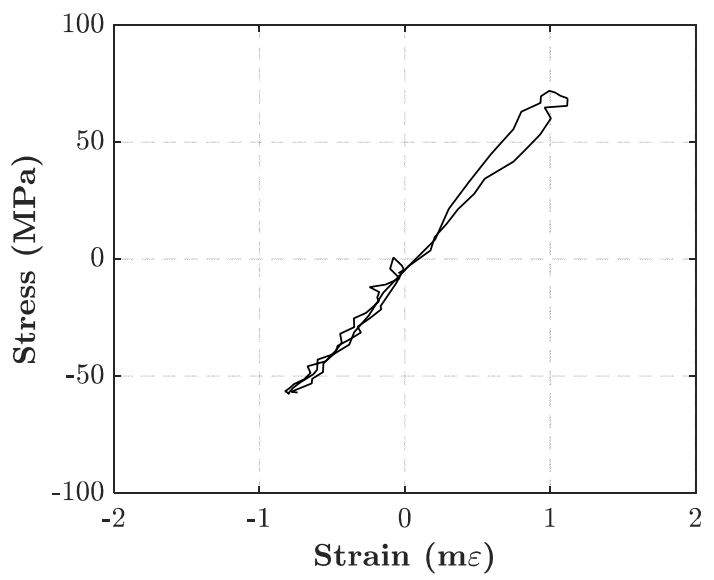

(c)

Fig. 8. Processed data for test Alu6 (clear focusing, 1 Mfps, 5 pixels per period, virtual gauge size $=10 \mathrm{~mm}, n=2$ ). (a) Strain in the cross-section at $40 \mathrm{~mm}$ from the free surface versus time obtained from the grid method compared to the data from the strain gauge, (b) stress in the cross-section at $50 \mathrm{~mm}$ from the free surface versus time obtained by the VFM method 
compared to the experimental strain multiplied by the sample Young's modulus, (c) stress-strain curve in the cross-section at $50 \mathrm{~mm}$ from the free end.

Table 2. Identified values of the Young's modulus for the test Alu6 by performing the linear regression of the stress-strain curves at $30 \mathrm{~mm}, 40 \mathrm{~mm}$ and $50 \mathrm{~mm}$ from the free surface (virtual gauge size $20 \mathrm{~mm}$ ).

\begin{tabular}{|l|l|l|}
\hline Virtual gauge position $(\mathrm{mm})$ & $\begin{array}{l}\text { Young's Modulus } E_{\text {lin. }} \\
(\mathrm{GPa})\end{array}$ & $\sigma_{\text {res }}^{\text {est }}(\mathrm{MPa})$ \\
\hline 30 & 65.3 & 5.5 \\
\hline 40 & 69.6 & 4.6 \\
\hline 50 & 74.5 & 6.7 \\
\hline
\end{tabular}

\section{Influence of processing parameters on stress and strain}

The influence of processing parameters used to filter out noise on the reconstructed stress from acceleration is now discussed. The stress level in a given cross-section of the field view is given as a function of time (or number of frames) according to Equation 6. A temporal filtering is applied in order to derive the time resolved acceleration [Savitzky and Golay 1964].

The effect of the polynomial function degree $(n)$ fitted over displacements over a time window with a length $(I)$ is now discussed. The results obtained from processing Alu6experiment with varying polynomial degree from 2 to 6 are detailed in the Figure 9 while ' $l$ ' was kept constant at 11 images (i.e. $11 \mu \mathrm{s}$ ). It can be observed that high polynomial degree tends to produce enhanced fluctuations. Finally a degree 2 polynomial function has been considered in the processing of the data, though degree 3 provides similar performance and could also have been selected.
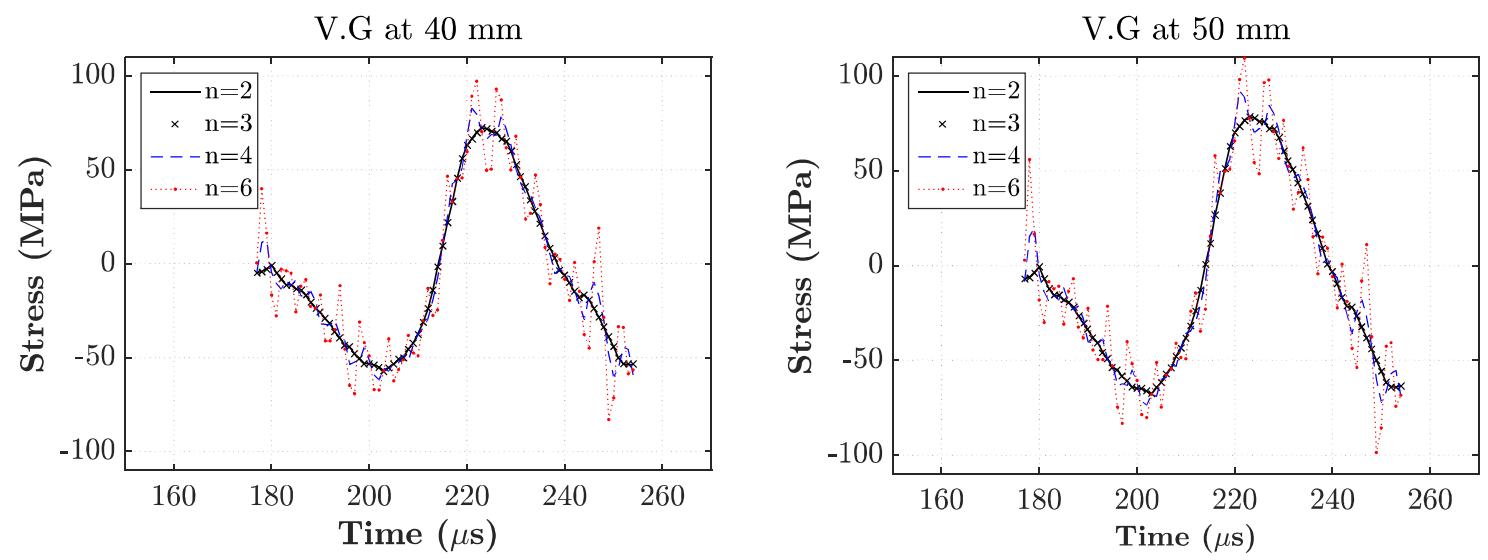

Fig. 9. Influence of the polynomial degree (n) used in processing the data of test Alu6 and deriving the acceleration fields (clear focusing, $1 \mathrm{Mfps}, 5$ pixels per period, window length $11 \mu \mathrm{s}$ ).

Next, the effect of the time window length ' $l$ ' is examined while keeping the $2^{\text {nd }}$ order polynomial. In Figure 10, several values of consecutive frames forming the size of the time window ' $l$ ' are considered to plot the stress in a given cross-section as a function of time. It is observed that for ' $l$ ' equal to 11 and 13 , a very similar response is obtained, without the higher order fluctuations obtained with lowed values. Finally, a value of ' $l$ ' equal to 11 was used for interframe-time equal to $1 \mu$ s whereas ' $l$ ' was set to 7 for the interframe-time of $2 \mu \mathrm{s}$, so the time window length does not exceed $14 \mu \mathrm{s}$. 

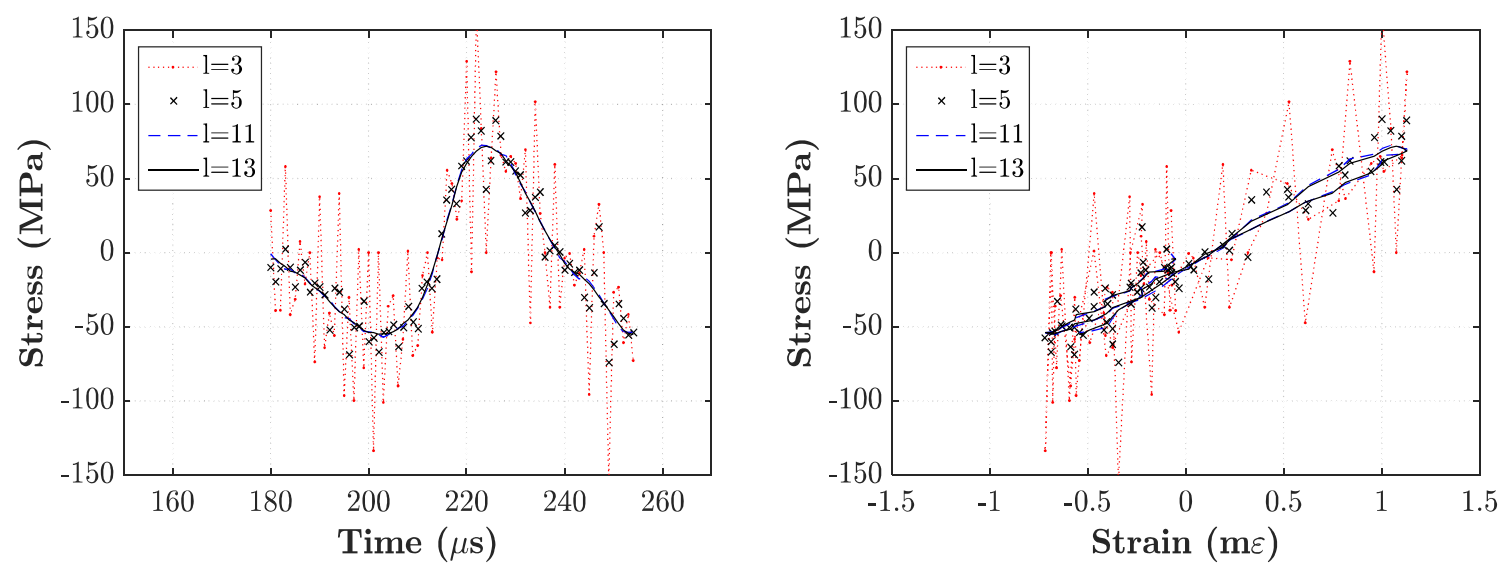

Fig. 10. Influence of the number of consecutive frames used for window length ' $l$ ' in processing the data of test Alu6 (clear focusing, $1 \mathrm{Mfps}, 5$ pixels per period, VG at $40 \mathrm{~mm}$ distance with spatial size of $20 \mathrm{~mm}$ ).

The effect of spatial size of the virtual gauge (VG) was also investigated and is plotted in Figure 11. The stress is calculated by averaging the value of stress provided by the VFM over $10 \mathrm{~mm}, 20 \mathrm{~mm}$ and $30 \mathrm{~mm}$. It is observed that with the considered grid pitch (1 $\mathrm{mm}$ ), the spatial filtering has a very small influence on the stress versus time curve. On the other hand, spatial filtering of $20 \mathrm{~mm}$ has a slight positive effect on the strain. Finally spatial filtering over $20 \mathrm{~mm}$ has been considered to process the experimental data in the next section. In conclusion, the parameters selected to process the data of the five spalling experiments performed with a $1 \mathrm{~mm}$ pitch grid are summarized in the Table 3 .
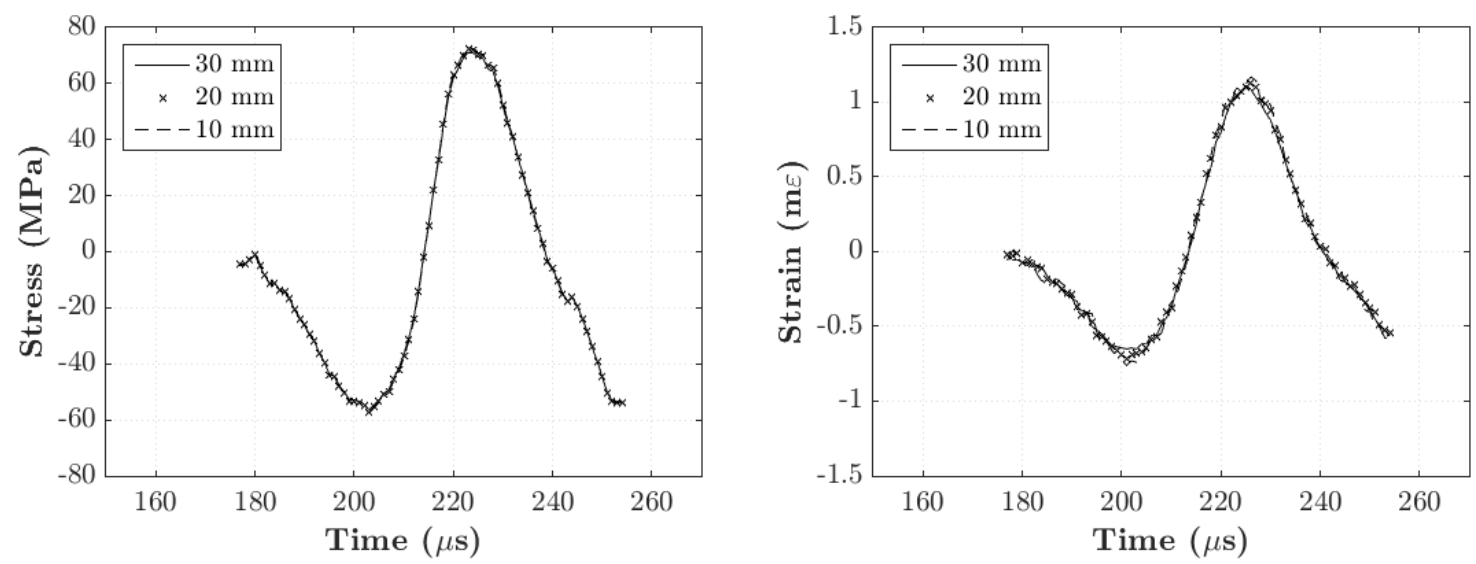

Fig. 11. Influence of the length of spatial averaging 'virtual gauge length' for processing the data of the Alu6 test (clear focusing, $1 \mathrm{Mfps}, 5$ pixels per period).

Table 3. Selected imaging parameters for the 5 tested samples with the HPV-1 camera

\begin{tabular}{|l|l|l|l|l|l|}
\hline Tests & $\begin{array}{l}\text { Interframe } \\
\text { time }\end{array}$ & 'n', 'l' & Focusing & $\begin{array}{l}\text { Interframe } \\
\text { time }\end{array}$ & Pixels/period \\
\hline Alu6 & $1 \mu \mathrm{s}$ & 2,11 & clear & $1 \mu \mathrm{s}$ & 5 \\
\hline Alu7 & $2 \mu \mathrm{s}$ & 2,7 & clear & $2 \mu \mathrm{s}$ & 5 \\
\hline Alu13 & $1 \mu \mathrm{s}$ & 2,11 & blurred & $1 \mu \mathrm{s}$ & 4 \\
\hline Alu8 & $1 \mu \mathrm{s}$ & 2,11 & blurred & $1 \mu \mathrm{s}$ & 5 \\
\hline Alu9 & $2 \mu \mathrm{s}$ & 2,7 & blurred & $2 \mu \mathrm{s}$ & 5 \\
\hline
\end{tabular}




\section{Influence of testing parameters on the measurement of stress and strain}

Five tests have been conducted on aluminium alloy samples with different imaging configurations, as listed in Table 2. The following parameters have been studied: interframe time, number of pixels per period and focusing. In tests 6,13 and 8 the recording frequency was set to the maximum value available with the HPV-1 Shimadzu camera ( $1 \mathrm{Mfps}$ ). The total recording time is equal to $102 \mu \mathrm{s}$. However, at such recording speed a significant increase of noise is observed with the HPV-1 camera [Rossi et al, 2013]. It is the reason why in two tests the recording frequency was set to $500 \mathrm{kfps}$ (interframe time equal to $2 \mu \mathrm{s}$ ). In addition, in four tests the size of the field of view was adjusted to get 5 pixels sampling each period of the grid. However, in test Alu13, the field of view was set to have only 4 pixels per period. This can be considered as the minimum value that can reasonably be used with the grid method and has shown some decent results in the past [Xavier et al, 2009], the theoretical lowest sampling being three pixels. Regarding the low fill-factor of the HPV-1 Shimadzu camera, defocusing can improve measurements as it filters out the high spatial frequency contents of the grid signal [Rossi et al, 2013]. Some positive effects of blurring were also suggested using simulated dynamic experiments in [Lukić et al, 2017]. Consequently, three tests were conducted by defocusing the lens so the grid appears as blurred exhibiting a quasisinusoidal profile. Distributions of grey levels are plotted in Figure 12 for the 5 experiments. Wider distributions of grey levels are observed in the case of blurred compared to clear pictures. It should be noted that in all the experiments, the lighting was adjusted to ensure grey level values not exceeding 70 as this is paramount to controlling the image quality with this camera [Rossi et al, 2013].

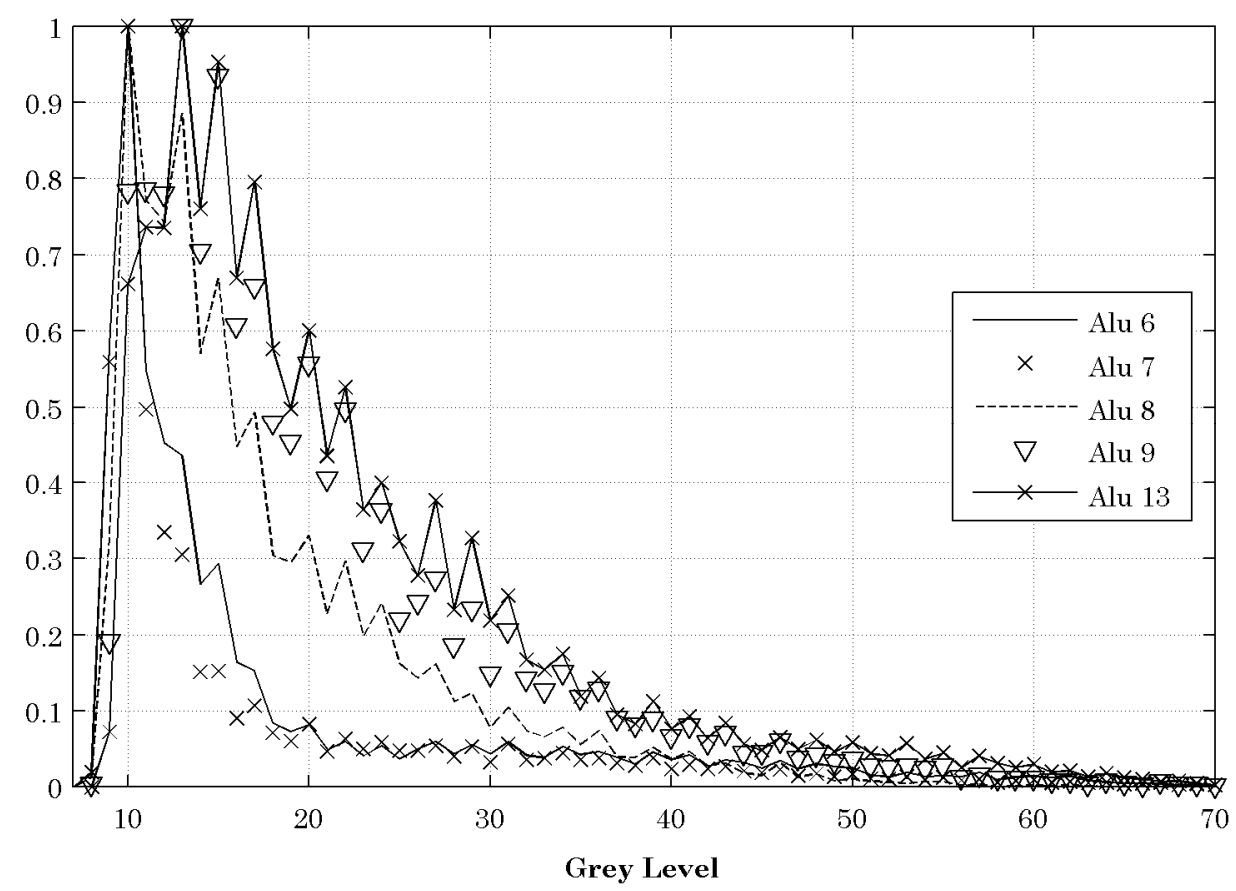

Fig. 12. Image grey level distributions recorded with the Shimadzu HPV-1 camera for the 5 samples normalized by the peak value. 
Figure 13 shows the results for test Alu7 (clear focusing, 0.5 Mfps, 5 pixels per period). Again, the stress in the cross-section at $50 \mathrm{~mm}$ from the free surface is plotted as a function of time in Figure 13a (Equation 6). The curve is compared to the data obtained from the strain gauge $(\mathrm{E} \times \varepsilon)$. Again, a good correlation is observed between both measurements. The stress-strain curve in the cross-section at $50 \mathrm{~mm}$ from the free surface is plotted in Figure 13b. The behaviour looks linear elastic even though a fair amount of noise is present. This noise is higher than for sample Alu6 (1Mfps). The maximum difference relative to the expected stress-strain elastic response is about $\pm 15 \mathrm{MPa}$ in stress and $\pm 2.10^{-4}$ in strain level. The identified modulus value from linear regression at three characteristic distances is provided in Table 4. Only the first compressive and tensile stages of the loading are considered for the regression and a virtual gauge size of $20 \mathrm{~mm}$ was used.

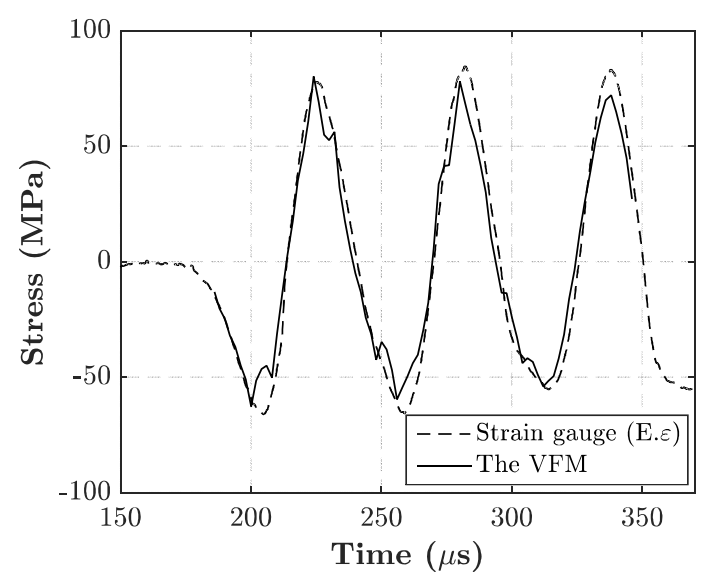

(a)

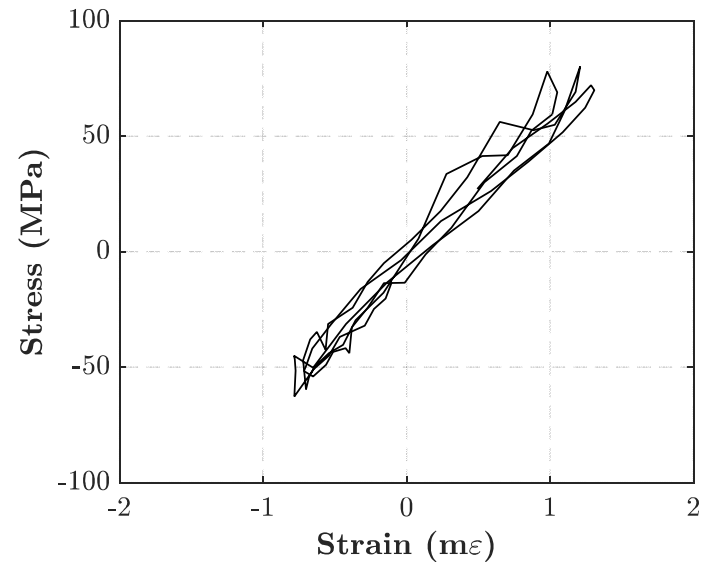

(b)

Fig. 13. Results for test Alu7 (clear focusing, $0.5 \mathrm{Mfps}, 5$ pixels per period). (a) Stress in the cross-section at $50 \mathrm{~mm}$ from the free surface versus time, (b) stress-strain curve in the crosssection at $50 \mathrm{~mm}$ from the free end.

Table 4. Identified values of the Young's modulus for the test Alu7 by performing linear regression onf the stress-strain curves at $30 \mathrm{~mm}, 40 \mathrm{~mm}$ and $50 \mathrm{~mm}$ from the free surface.

\begin{tabular}{|l|l|l|}
\hline Virtual gauge position $(\mathrm{mm})$ & $\begin{array}{l}\text { Young's Modulus } E_{\text {lin. }} \\
(\mathrm{GPa})\end{array}$ & $\sigma_{\text {res }}^{\text {est }}(\mathrm{MPa})$ \\
\hline 30 & 61.7 & 7.1 \\
\hline 40 & 61.8 & 11.1 \\
\hline 50 & 63.0 & 15.2 \\
\hline
\end{tabular}

The influence of blurring is analysed by comparing Figures 10 and 14. Figure 14 provides the data obtained by defocussing the lens of the camera (Alu8 test, blurred focusing, $1 \mathrm{Mfps}, 5$ pixels per period). Again, the stress in the cross-section at $50 \mathrm{~mm}$ from the free surface (Equation 6) is compared to the data obtained from the strain gauge $\left(\begin{array}{lll}\mathrm{E} & \mathrm{E}\end{array}\right)$ and a good correlation is observed between both measurements. The stress-strain curve in the cross-section at $50 \mathrm{~mm}$ from the free surface is plotted in Figure $14 \mathrm{~b}$. The quality of the curve is better than for the previous case but similar to that for clear focusing in Figure 8, with typical error about $7 \mathrm{MPa}$ in stress and $1.10^{-4}$ in strain compared to the line of slope 'E'. The obtained Young's modulus from linear regression at three characteristic distances is provided in Table 5. Only the first compressive and tensile stages of the loading are considered for the regression and a 
virtual gauge size of $20 \mathrm{~mm}$ is used. Finally blurred focusing is seen to have little influence on the quality of the measurements, the lowering the frame rate seems to affect the results much more.

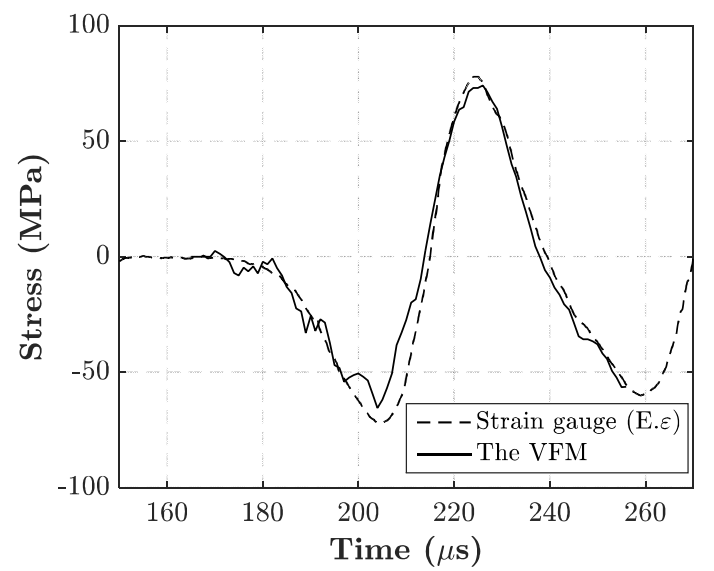

(a)

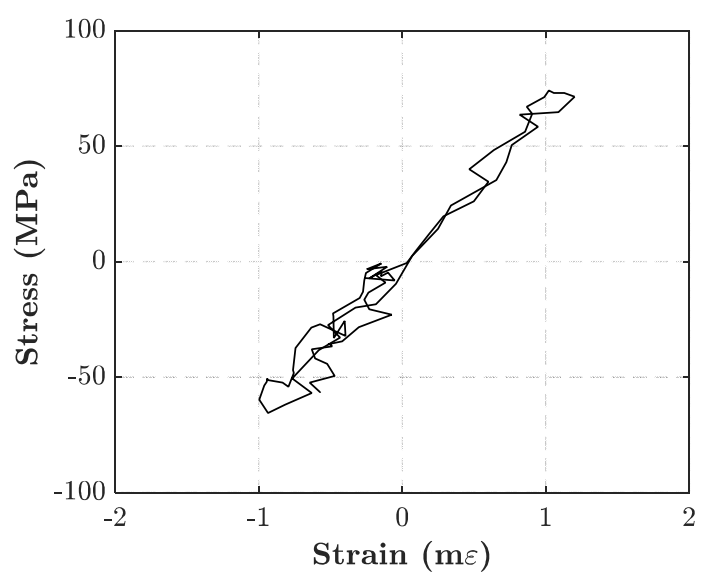

(b)

Fig. 14. Results test Alu8 (blurred focusing, 1 Mfps, 5 pixels per period). (a) Stress in the crosssection at $50 \mathrm{~mm}$ from the free surface versus time, (b) stress-strain curve in the cross-section at $50 \mathrm{~mm}$ from the free end.

Table 5. Identified values of the Young's modulus for the test Alu8 by performing the linear regression of the stress-strain curves at $30 \mathrm{~mm}, 40 \mathrm{~mm}$ and $50 \mathrm{~mm}$ from free surface.

\begin{tabular}{|l|l|l|}
\hline Virtual gauge position $(\mathrm{mm})$ & $\begin{array}{l}\text { Young's Modulus } E_{\text {lin. }} \\
(\mathrm{GPa})\end{array}$ & $\sigma_{\text {res }}^{\text {est }}(\mathrm{MPa})$ \\
\hline 30 & 63.7 & 6.7 \\
\hline 40 & 66.2 & 6.2 \\
\hline 50 & 69.9 & 8.2 \\
\hline
\end{tabular}

Experimental results obtained with blurred focusing at $0.5 \mathrm{Mfps}$ and with 5 pixels per period (test Alu9) are given in Figure 15. The obtained Young's modulus from linear regression at three characteristic distances is provided in Table 6. Only the first compressive and tensile stages of the loading are considered for the regression and a virtual gauge size of $20 \mathrm{~mm}$ was used. Again, a higher level of error is noted at $0.5 \mathrm{Mfps}$ compared to the experiment performed at $1 \mathrm{Mfps}$ (test Alu8, Figure 14). The difference relative to the expected stress-strain elastic response is about $\pm 12 \mathrm{MPa}$ in stress and $\pm 2.10^{-4}$ in strain. 


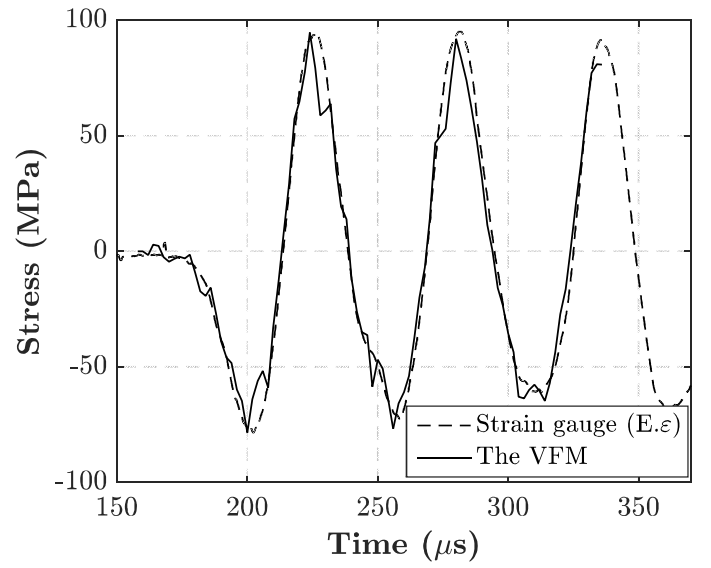

(a)

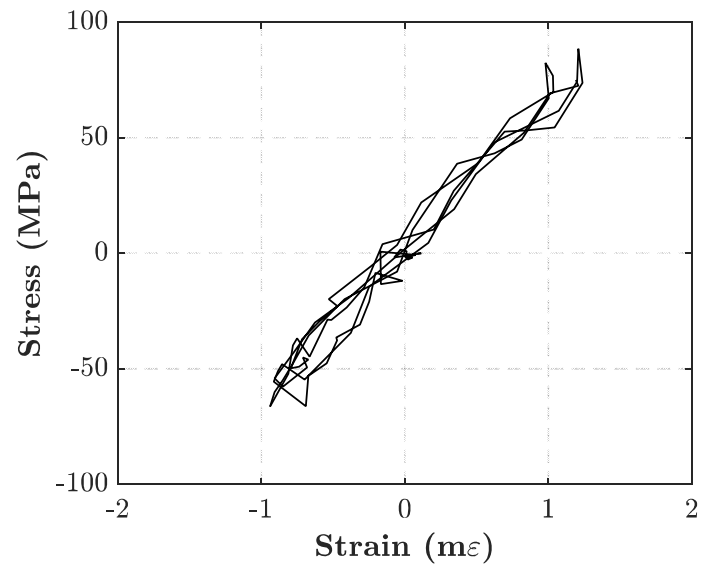

(b)

Fig. 15. Results for test Alu9 (blurred focusing, $0.5 \mathrm{Mfps}, 5$ pixels per period). (a) Stress in the cross-section at $40 \mathrm{~mm}$ from the free surface versus time, (b) stress-strain curve in the crosssection at $40 \mathrm{~mm}$ from the free surface.

Table 6. Identified values of the Young's modulus for the test Alu9 by performing the linear regression of the stress-strain curves at $30 \mathrm{~mm}, 40 \mathrm{~mm}$ and $50 \mathrm{~mm}$ from free surface.

\begin{tabular}{|l|l|l|}
\hline Virtual gauge position $(\mathrm{mm})$ & $\begin{array}{l}\text { Young's Modulus } E_{\text {lin. }} \\
(\mathrm{GPa})\end{array}$ & $\sigma_{\text {res }}^{\text {est }}(\mathrm{MPa})$ \\
\hline 30 & 62.7 & 7.4 \\
\hline 40 & 66.7 & 7.7 \\
\hline 50 & 67.4 & 11.4 \\
\hline
\end{tabular}

The influence of the spatial sampling of the grid is examined in Figure 16 by adjusting the field of view to have only 4 pixels per period (Alu13) instead of 5 pixels per period (Alu8, Figure 14). In the Alu13 experiment, the other testing parameters are set as in the Alu8 experiment (blurred focusing, $1 \mathrm{Mfps}$ ). It is observed that with 4 pixels per period, the level of error is increasing dramatically compared to 5 pixels per period, and provides a nonsensical stress-strain curve.
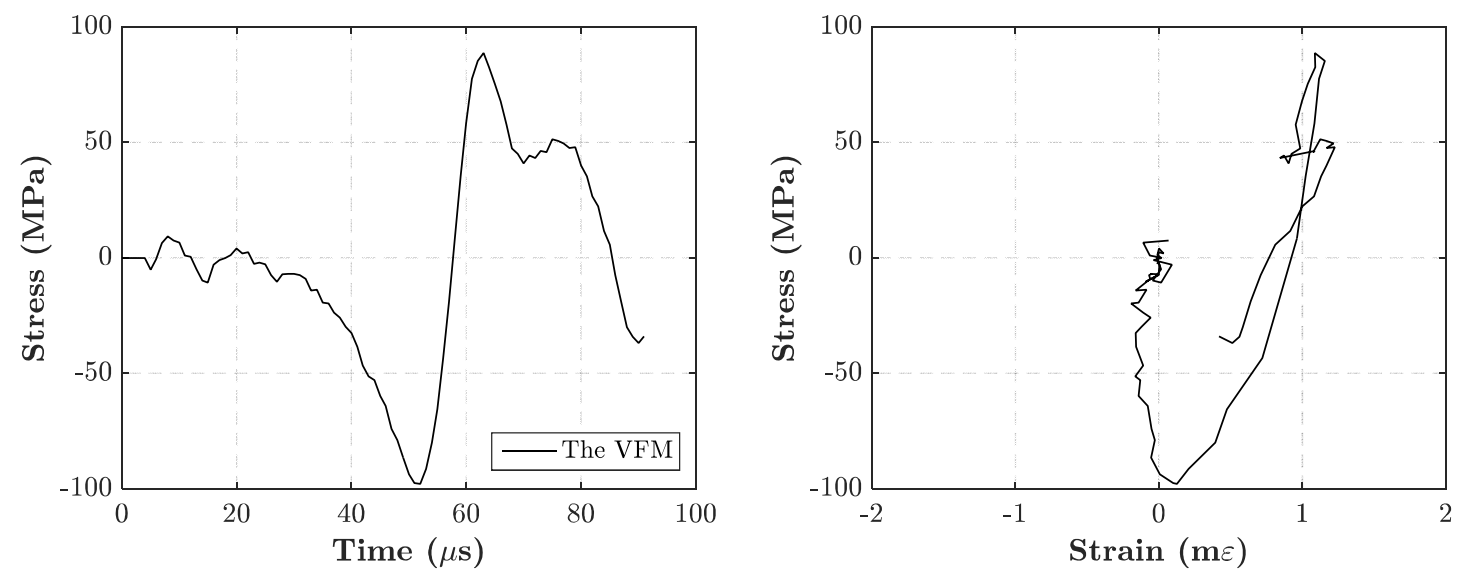

Fig. 16. Results for test Alu13 (blurred focusing, 1 Mfps, 4 pixels per period). Stress-strain curve in the cross-section at $60 \mathrm{~mm}$ from the free surface. 
In conclusion to this section, spalling experiments performed on aluminium alloy samples have shown a weak influence of the focusing. Experiments performed by slightly blurring the picture without excessive blurring seem to provide better results. However, a strong influence of the spatial sampling of the grid is noted between 4 and 5 pixels per period. Indeed, the test performed with 4 pixels per period enables a larger field of view to be recorded but the quality of the results sharply decreases. Even though satisfactory results were obtained in [Xavier et al, 2009] with this configuration, the strains are much smaller here and the fill factor of the camera much lower, both issues contributing to the sharp loss of quality. Finally, it is better to increase the recording frequency to the maximum level provided by the HPV-1 camera (1Mfps) as results at half this frame rate showed significantly degraded results. This is thought to be caused by the resolution on acceleration but would need to be confirmed.

\section{Experimental results obtained with a Kirana ultra-high speed camera}

A spalling test was carried on the same aluminium sample as in the previous section with a Specialized Imaging Ltd. Kirana camera. Due to high pixel count of captured frames, the grid pitch sampling was set to 13 pixels per grid period and the test was performed at $2 \mathrm{Mfps}$ with exposure of $300 \mathrm{~ns}$. The camera provides 180 images which correspond to $90 \mu$ s total recording time at that frame rate. The camera is equipped with a fast shutter which aims at reducing parasitic effects [Benhammadi et al, 2017]. This requires a use of a high power light source in order to illuminate the scene appropriately. Consequently, flash lights were used which were triggered using a short circuit rig placed at the bar-projectile contact. Due to the particular sensor technology, a certain high frequency oscillation is present within the sequence of acquired images (Figure 17). In order to reduce the peak grey levels oscillations on each 4 frames within a sequence of 10, these 4 images were removed and substituted with weighted average frames. An example of the effect of such correction is presented in Figure 17.

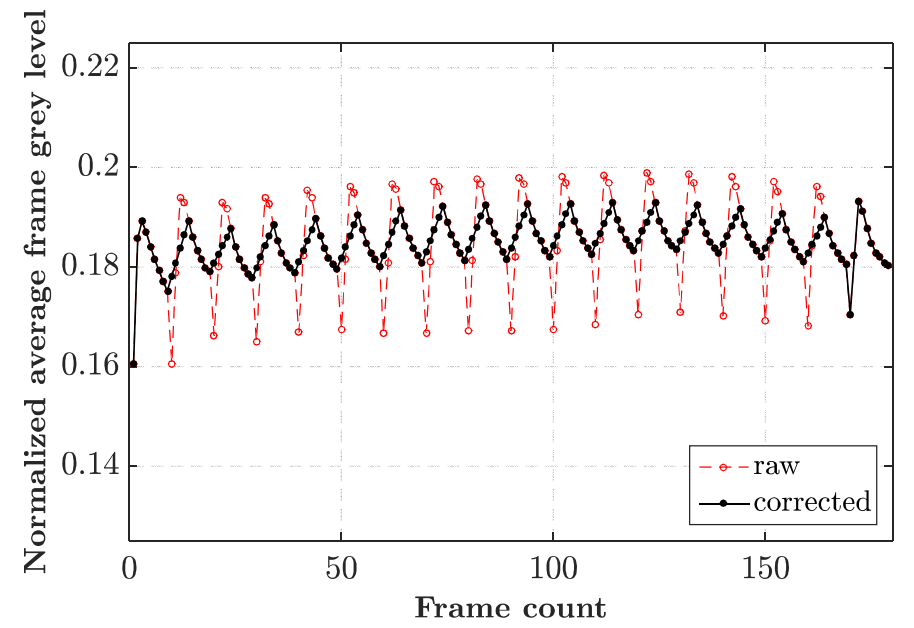

Fig. 17. Average frame grey level normalised by camera dynamic range for two series of images from the Kirana camera, with and without removed 'peak frames'.

Figure 18 depicts the results of the test. The same processing parameters were used to derive the deformation measurements from the measured displacement fields as in the previous section with $(n=2, l=15)$. The obtained Young's modulus from linear regression at four characteristic distances is provided in Table 7. Only the compressive 
and tensile stages of the loading are considered for regression and a virtual gauge size of $20 \mathrm{~mm}$ was used.

According to the experimental data of Figure 18, it is observed that without additional post-treatment of the images, the stress can deviate by more than $10 \mathrm{MPa}$ from the expected values derived from the strain gauge readings. In addition, the oscillating noise is characterised by a time period of 10 frames ( $5 \mu \mathrm{s}$ in the Figure 18) that precisely corresponds to the flickering frequency noted in Figure 17. Despite the temporal smoothing algorithm used in the data processing, the flickering in grey level is found in the processed results. However, the obtained Young's modulus from linear regression at four characteristic distances (Table 7) is just slightly overestimating the expected value of the tested aluminium alloy (72.8 GPa). This is because the oscillations in Figure 18a are superimposed to the correct low frequency carrier and are filtered out during the regression. However, the quality of the stress-strain curve is significantly lower.
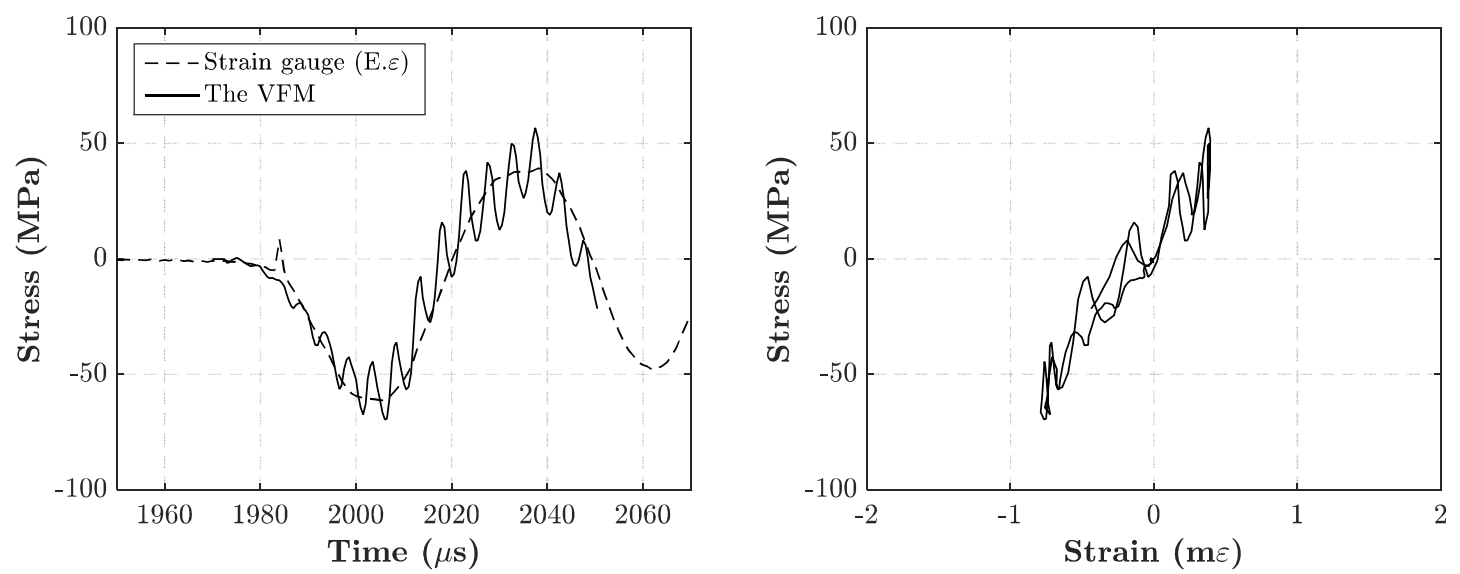

Fig. 18. Results of test 1803 with the Kirana camera (blurred focusing, 2 Mfps, 13 pixels per period). (a) Stress in the cross-section at $60 \mathrm{~mm}$ from the free surface versus time, (b) stressstrain curve in the cross-section at $60 \mathrm{~mm}$ from the free surface.

Table 7. Identified values of the Young's modulus for test 1803 by performing linear regression of the stress-strain curves at $30 \mathrm{~mm}, 40 \mathrm{~mm}, 50 \mathrm{~mm}$ and $60 \mathrm{~mm}$ from the free surface.

\begin{tabular}{|l|l|l|}
\hline Virtual gauge position $(\mathrm{mm})$ & $\begin{array}{l}\text { Young's Modulus } E_{\text {lin. }} \\
(\mathrm{GPa})\end{array}$ & $\sigma_{\text {res }}^{\text {est }}(\mathrm{MPa})$ \\
\hline 30 & 80.2 & 6.2 \\
\hline 40 & 86.5 & 8.9 \\
\hline 50 & 84.7 & 10.1 \\
\hline 60 & 79.6 & 10.4 \\
\hline
\end{tabular}

6. Experimental results obtained with a Shimadzu HPV-X2 ultra-high speed camera

In order to investigate the performance of an another CMOS-based ultra-high speed imaging system, a spalling test on an aluminium alloy was carried out using the above described Shimadzu HPV-X2 camera. The sensor uses the FTCMOS2 chip technology which differs from the previous generation having a much higher sensitivity to light 
thanks to improved voltage transfer [Kuroda et al, 2016]. This allows for the use of a continuous light source even at interframe times responding to $200 \mathrm{~ns}$.

The results obtained from a spalling test performed on an aluminium alloy using the Shimadzu HPV-X2 camera are depicted on Figure 19. The same processing parameters were used to derive the deformation measurements from the measured displacement fields as in the previous section $(n=2, l=15)$. Finally, the evolution of stress versus time and stress versus strain is plotted on Figure 19. A good match is observed with the response provided by the strain gauge (Figure 19a). The obtained Young's modulus from the linear regression at three characteristic distances is provided in Table 8. The obtained value of Young's modulus is underestimated by only 7\%.

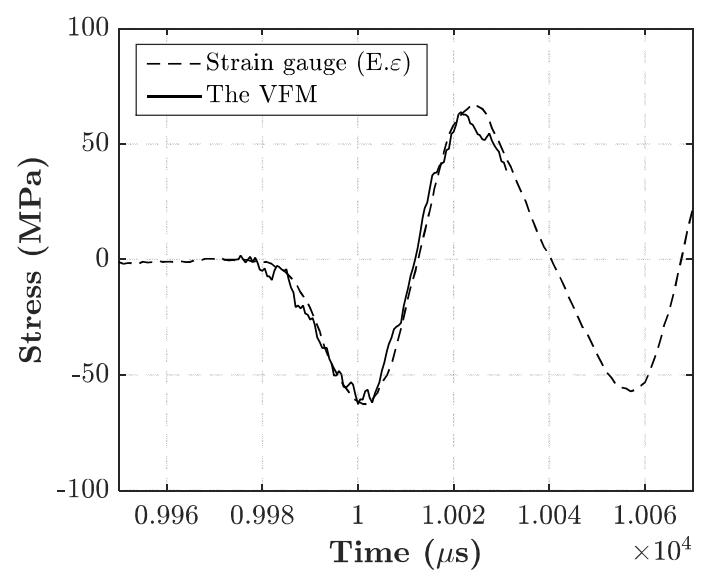

(a)

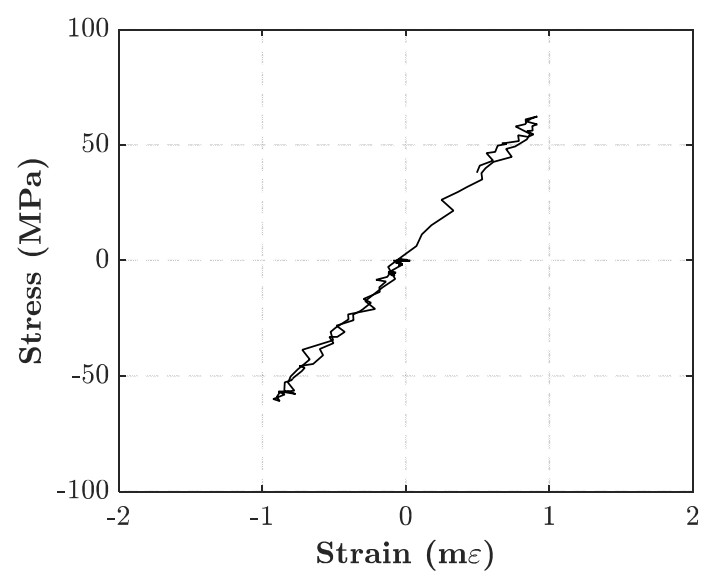

(b)

Fig. 19. Results for test 1703 with the Shimadzu HPV-X2 camera (blurred focusing, 2 Mfps, 7 pixels per period). (a) Stress in the cross-section at $40 \mathrm{~mm}$ from the free surface versus time, (b) stress-strain curve in the cross-section at $40 \mathrm{~mm}$ from the free surface.

Table 8. Identified values of the Young's modulus for test 1703 by performing the linear regression of the stress-strain curves at $30 \mathrm{~mm}, 40 \mathrm{~mm}$ and $50 \mathrm{~mm}$ from the free surface.

\begin{tabular}{|l|l|l|}
\hline Virtual gauge position $(\mathrm{mm})$ & $\begin{array}{l}\text { Young's Modulus } E_{\text {lin. }} \\
(\mathrm{GPa})\end{array}$ & $\sigma_{\text {res }}^{\text {est }}(\mathrm{MPa})$ \\
\hline 30 & 69.1 & 2.9 \\
\hline 40 & 67.9 & 4.2 \\
\hline 50 & 67.8 & 5.1 \\
\hline
\end{tabular}

\section{Conclusion}

Spalling experiments have been performed on aluminium alloy samples covered by a flat grid imaged with three types of ultra-high speed camera technologies in order to evaluate the influence of several testing parameters, processing parameters and sensor technologies on the results. The processing method relies, on the one hand, on pointwise measurements of data recorded with strain gauges, and, on the other hand, on the time resolved measurement of axial displacement fields by means of images from one of these ultra-high speed cameras and the Virtual Fields Methods (VFM). The acceleration fields that are derived from the obtained displacement maps provided the average axial 
stress in any cross-section within the field of view, so the local stress-strain response can be identified.

First, a series of spalling experiments have been conducted with the Shimadzu HPV-1 camera to investigate the effect of three processing parameters (degree of fitted polynomial function and number of consecutive frames for temporal filtering, and kernel of spatial filtering) on the results. It was noted that 'reasonable' temporal filtering of the acceleration field and spatial filtering of strain field allowed a significant improvement of the measured data. Temporal filtering of the acceleration field is particularly needed to decrease the effect of noise resulting from the double temporal differentiation of the displacement field.

Then, the effects of three testing parameters (interframe time, focusing, number of pixels per period) have been analysed. These experiments have shown a weak influence of the focusing. Sharp or slightly blurred images seemed to provide the best results. In addition, a strong influence of the number of pixel per period was noted. Indeed, if a grid sampling of 4 pixels per period allows to record data over a larger field of view, the quality of the results is strongly compromised. This may be caused by the low fill factor of the camera as this sampling has been used in the past with standard CCD cameras and provided more reasonable quality. In addition, it is better to increase the recording frequency to the maximum level provided by the HPV-1 camera (1Mfps).

Following this, two additional ultra-high speed cameras that provide increased spatial resolution and recording frame-rate were employed to investigate the benefits in terms of measurement quality brought by CMOS based sensor technology. Spalling experiments on the same aluminium alloy sample were conducted with the Specialized Imaging Ltd. Kirana and the Shimadzu HPV-X2 cameras. The first camera provides superior number of pixels and number of stored frames that can be an advantage, respectively, for improving the spatial resolution of full-field measurements and the total recording time (at a given recording frequency). However the processed data show an oscillating noise concerning the stress versus time response that results from the noise in the acceleration fields due to grey level flickering.

Finally, this work shows that the spalling set-up applied to a sample of known characteristics (aluminium alloy in the present case) can be a good benchmark to evaluate the performances of UHS cameras and evaluate the effect of experimental and processing parameters.

\section{Acknowledgments}

Professor F. Pierron gratefully acknowledges funding through EPSRC grant EP/L026910/1. This work has been partially funded by the LabEx Tec 21 (Investissements d'Avenir - grant agreement nANR-11-LABX-0030) from the Univ. Grenoble Alpes. The provided support is gratefully acknowledged by the authors. Professor P. Forquin gratefully acknowledges the LMT-Cachan Laboratory for providing the Shimadzu HPV-X2 camera used in the present work.

\section{Bibliography}

Aloui, S., Othman, R., Poitou, A., Guégan, P., El-Borgi, S., 2008. Non-parametric identification of the nonhomogeneous stress in high strain-rate uni-axial experiments. Mechanics Research Communications, 35(6):392-397. 
Avril, S., Bonnet, M., Bretelle, A.-S., Grédiac, M., Hild, F., Ienny, P., Latourte, F., Lemosse, D., Pagano, S., Pagnacco, E., Pierron, F., 2008. Overview of identification methods of mechanical parameters based on full-field measurements. Experimental Mechanics, 48(4):381-402.

Avril, S., Ferrier, E., Hamelin, P., Surrel, Y., Vautrin, A., 2004. A full-field optical method for the experimental analysis of reinforced concrete beams repaired with composites. Composite Part A, 35(78):873-884.

Avril, S., Feissel, P., Pierron, F., Villon, P., 2010. Comparison of two approaches for controlling the uncertainty in data differentiation: application to full-field measurements in solid mechanics, Measurement Science and Technology, 21, 015703 (11 pp).

Badulescu, C., Grédiac, M., Haddadi, H., Mathias, J. D., Balandraud, X., Tran, H. S., 2011. Applying the grid method and infrared thermography to investigate plastic deformation in aluminium multicrystal. Mechanics of Materials, 43(1):36-53.

Bedsole, R., Tippur, H. V., 2013. Dynamic fracture characterization of small specimens: a study of loading rate effects on acrylic and acrylic bone cement. Journal of Engineering Materials and Technology, 135(3):031001.

Benhammadi, S., Marsh, B., Taylor, K., Chan, W., Birman, A., Lahav, A., Fenigstein, A., Turchetta, R., 2017. A 5 Million fps Global Shutter Megapixel Sensor with Shutter Efficiency in Excess of 120 dB. In 2017 International Image Sensor Workshop, pages 195-198.

Besnard, G., Hild, F., Lagrange, J. M., Martinuzzi, P., Roux, S., 2012. Analysis of necking in high speed experiments by stereocorrelation. International Journal of Impact Engineering, 49:179-191.

van Blitterswyk, J., Fletcher, L., Pierron, F., 2018. Image-based inertial impact test for composite interlaminar tensile properties. Journal of Dynamic Behavior of Materials. In press. Doi: 10.1007/s40870018-0175-1.

Cadoni, E., Albertini, C., Solomos, G., 2006. Analysis of the concrete behaviour in tension at high strain-rate by a modified Hopkinson bar in support of impact resistant structural design. Journal of Physics IV, 134:647-652.

Crooks, J., Marsh, B., Turchetta, R., Taylor, K., Chan, W., Lahav, A., Fenigstein, A., 2013a. Kirana: a solid-state megapixel uCMOS image sensor for ultrahigh speed imaging. In Widenhorn, R. and Dupret, A., editors, Sensors, Cameras, and Systems for Industrial and Scientific Applications XIV, Volume 8659, page 865903.

Crooks, J., Marsh, B., Turchetta, R., Taylor, K., Chan, W., Lahav, A., Fenigstein, A., 2013b. Ultra-high speed imaging at megaframes per second with a megapixel CMOS image sensor. International Image Sensor Workshop, pages 4-7.

Erzar, B., Forquin, P., 2010. An experimental method to determine the tensile strength of concrete at high rates of strain. Experimental MechAnics. 50(7):941-955.

Erzar, B., Forquin, P., 2014. Analysis and modelling of the cohesion strength of concrete at high strainrates. International Journal of Solids and Structures.51(14):2559-2574.

Erzar, B., Forquin, P., 2011. Experiments and mesoscopic modelling of dynamic testing of concrete. Mechanics of Materials. 43:505-527.

Etoh, T.G., Poggemann, D., Kreider, G., Mutoh, H., Theuwissen, A.J.P., Ruckelshausen, A., Kondo, Y., Maruno, H., Takubo, K., Soya, H., Takehara, K., Okinaka, T., Takano, Y., 2003. An image sensor which captures 100 consecutive frames at 1000000 frames/s. IEEE Transactions on Electron Devices, 50(1):144-151.

Fletcher, L., Pierron, F., 2018. An image-based inertial impact (IBII) test for tungsten carbide cermets. Journal of Dynamic Behavior of Materials, 4(4):481-504.

Forquin, P., Erzar, B., 2010. Dynamic fragmentation process in concrete under impact and spalling tests. International Journal of Fracture. 163:193-215.

Forquin, P., Sallier, L., Pierron, F., 2013. On the use of the Virtual Fields Method to characterize the dynamic response of geomaterials in spalling tests. DYMAT Technical meeting, London, UK.

Forquin, P., Riedel, W., Weerheijm, J., 2013. Chapter 6: Dynamic test devices for analysing the tensile properties of concrete, in Tensile strength of concrete, published by Woodhead Publishing. 
Forquin, P., Hild, F., 2010. A probabilistic damage model of the dynamic fragmentation process in brittle materials. Advances in Applied Mechanics. Giessen \& Are feds. 44:1-72. Academic Press, San Diego, CA.

Forquin, P., Lukić, B., 2018. On the processing of spalling experiments. Part I: Identification of the dynamic tensile strength of concrete. J. Dyn. Behavior Materials. 4(1): 34-55

Frew, D. J., Forrestal, M. J., Chen, W., 2005. Pulse shaping techniques for testing elastic-plastic materials with a split Hopkinson pressure bar. Experimental Mechanics. 45 (2): 186-195.

Gilat, A., Schmidt, T. E., Walker, A. L., 2009. Full field strain measurement in compression and tensile split Hopkinson bar experiments. Experimental Mechanics. 49(2):291-302.

Grange, S., Forquin, P., Mencacci, S., Hild, F., 2008. On the dynamic fragmentation of two limestones using edge-on impact tests. International Journal of Impact Engineering. 35:977-991.

Grantham, S. G., Siviour, C. R., Proud, W. G., Field, J. E., 2004. High strain rate Brazilian testing of an explosive simulant using speckle metrology. Measurement Science and Technology. 15(9):1867-1870.

Grédiac, M., Sur, F., and Blaysat, B., 2016. The Grid Method for in-plane displacement and strain measurement: a review and analysis. Strain. 52(3):205-243.

Grédiac, M., Blaysat, B., Sur, F., 2017. A critical comparison of some metrological parameters characterizing local Digital Image Correlation and Grid Method. Experimental Mechanics, 57(6), 871-903. doi:10.1007/s11340-017-0279-x

Kajberg, J., Sundin, K. G., Melin, L. G., Ståhle, P., 2004. High strain-rate tensile testing and viscoplastic parameter identification using microscopic high-speed photography. International Journal of Plasticity. 20(4-5):561-575.

Klepaczko, J.R., Brara, A., 2001. An experimental method for dynamic tensile testing of concrete by spalling. International Journal of Impact Engineering. 25:387-409.

Kuroda, R., Tochigi Y., Miyauchi, K., Takeda, T., Sugo, H., Shao, F., Sugawa, S., 2016. A 20Mfps Global Shutter CMOS Image Sensor with Improved Light Sensitivity and Power Consumption Performances. ITE Trans. Media Tech. and Applications, 4: 149-154.

Lukić, B., 2018. Mise au point d'une technique de mesure de champs pour la caractérisation du comportement dynamique du béton en traction. PhD thesis. Communauté Université Grenoble Alpes, Grenoble, France.

Lukić, B., Saletti, D., Forquin, P., 2017. Use of simulated experiments for material characterization of brittle materials subjected to high strain rate dynamic tension. Philosophical Transactions of the Royal Society A, 375(2085): 20160168.

Lukić, B., Saletti, D., Forquin, P., 2018. On the processing of spalling experiments. Part II: Identification of Concrete Fracture Energy in Dynamic Tension. J. Dyn. Behavior Materials. 4(1):56-73.

Luo, H., Lu, H., Leventis, N., 2006. The compressive behavior of isocyanate-crosslinked silica aerogel at high strain rates. Mechanics of Time-Dependent Materials. 10(2):83-111.

Moulart, R., Rotinat, R., Pierron, F., Lerondel, G., 2007. On the realization of microscopic grids for local strain measurement by direct interferometric photolithography. Optics and Lasers in Engineering. 45(12):1131-1147.

Moulart, R., Pierron, F., Hallett, S. R., Wisnom, M. R., 2011. Full-field strain measurement and identification of composites moduli at high strain rate with the virtual fields method. Experimental Mechanics. 51(4):509-536.

Mutoh, H., Etoh T., 2003. European Patent Application: EP 1278246 A1, Int. application number: PCT/JP01/02531, Int. publication number: WO 01/073849 (04.10.2001 Gazette 2001/40).

Novikov, S.A., Divnov, I.I., Ivanov, A.G., 1966. The study of fracture of steel, aluminium and copper under explosive loading. Fizika Metallov i Metallovedeniye. 21: 608-615.

Peirs, J., Verleysen, P., Van Paepegem, W., Degrieck, J., 2011. Determining the stress-strain behaviour at large strains from high strain rate tensile and shear experiments. International Journal of Impact Engineering. 38(5):406-415. 
Pierron, F., Cheriguene, R., Forquin, P., Moulart, R., Rossi, M., Sutton, M.A., 2011. Performances and limitations of three ultra-high speed imaging cameras for full-field deformation measurements. Applied Mechanics and Materials. 70: 81-86.

Pierron, F., Forquin, P., 2012. Ultra high speed full-field deformation measurements on concrete spalling specimens and stiffness identification with the Virtual Fields Method. Strain. 48(5):388-405.

Pierron, F., Grédiac, M., 2012. The Virtual Fields Method, Springer New-York, ISBN 978-1-4614-1824-5.

Pierron, F., Sutton, M.A., Tiwari, V., 2011. Ultra high speed DIC and virtual fields method analysis of a three point bending impact test on an aluminium bar. Experimental Mechanics. 51(4):537-563.

Pierron, F., Zhu, H., Siviour, C., 2014. Beyond Hopkinson's bar, Philosophical Transactions of the Royal Society A. 372:20130195.

Pierron, F., 2018. http://photodyn.org/tools/ultra-high-speed-camera.

Piro, J.L., Grédiac, M., 2004. Producing and transferring low-spatial-frequency grids for measuring displacement fields with moiré and grid methods. Experimental Techniques. 28(4):23-26.

Reu, P.L., Miller, T.J., 2008. The application of high-speed digital image correlation. Journal of Strain Analysis for Engineering Design. 43(8):673-688.

Riedel, W., Forquin, P., 2013. Chapter 5: Modelling the response of concrete structures to dynamic loading, in Tensile strength of concrete, published by Woodhead Publishing.

Rossi, M., Pierron, F., Forquin, P., 2014. Assessment of the metrological performances of an ISIS ultra-high speed camera for full-field deformation measurements. Measurement Science and Technology. 25:(2): 025401.

Saadati, M., Forquin, P., Weddfelt, K., Larsson, P.L., Hild, F., 2014. Granite rock fragmentation at percussive drilling - experimental and numerical investigation. International Journal for Numerical and Analytical Methods in Geomechanics. 38: 828-843.

Savitzky, A., Golay, M., 1964. Smoothing and differentiation of data by simplified least squares procedures. Analytical Chemistry. 36(8):1627-1639.

Schuler, H., Mayrhofer, C., Thoma, K., 2006. Spall experiments for the measurement of the tensile strength and fracture energy at high strain rates. International Journal of Impact Engineering. 32:1635-1650.

Seghir, R., Pierron, F., 2018. A novel image-based ultrasonic test to map material mechanical properties at high strain-rates. Experimental Mechanics, 58(2), 183-206. doi:10.1007/s11340-017-0329-4.

Surrel, Y., 1994. Moiré and grid methods: a signal-processing approach. In R.J. Pryputniewicz and J. Stupnicki, editors, Interferometry '94 : Photomechanics, volume SPIE 2342.

Tedesco, J.,W., Ross, C.,A., Kuennen, S.,T., 1993., Experimental and numerical analysis of high strain-rate splitting tensile tests. ACI materials Journal, 92 (2): 162-169.

Weerheijm, J., Van Doormaal, J.C.A.M., 2007. Tensile failure of concrete at high loading rates: new test data on strength and fracture energy from instrumented spalling tests. International Journal of Impact Engineering. 34:609-626.

Xavier, J., Avril, S., Pierron, F., Morais, J., 2009. Variation of transverse and shear stiffness properties of wood in a tree. Composites Part A. 40(12):1953-1960. 